

\title{
A GENERAL-PURPOSE MICROPROCESSOR-BASED CONTROL CHASSIS
}

by

\author{
James K. Halbig, S. F. Klosterbuer, and Donaid A. Swenson
}

\begin{abstract}
The objective of the Pion Generation for Medical Irradiations (PIGMI) program at the Los Alamos Suientific Laboratory is to develop the technolosy to build manaller, less expensive, and more reliable proton linear accelerators for medical applications. For this program, we developed a powerful, simple, inexpenive, and reliable control and data acquisition system. The system has a NOVA $3 D$ computer with a real time diakoperating system (RDOS) that communicates with distribsted microprocessor-based controllers which directly control data input/output chassis. At the beart of the controller is a microprocessor crate which was conceived at the Fermi National Acrelerator Laboratory. We applied this idea to the design of the hardware and software of the controller.
\end{abstract}

\section{INTRODUCTION}

Microprocessors are used widely in research and development applications. Their value in accelerator control was sensed early at the Fermi National Accelerator Laboratory (FNAL) where an extremely adaptable, modular microprocessor-based controller was conceived. ${ }^{2}$

The main advantages of the controller are its modularity and interfaceability. For example, one 115- by $165-\mathrm{mm}$ board has 64 binary data lines and 16 handshake lines. These lines are divided into four identical ports. Each port has 16 binary data lines that can be programmed as inputs or outputs, and 4 handshake lines, 2 of which can be programmed as inputs or outputs and 2 that are inputs only. Peripheral devices interface to this board, called a peripheral interface adapter (PIA) board, via four 26-conductor ribbon cables that have "smash-on," solderless connectors.
The objective of the Pion Generation for Medical Irradiations (PIGMI) project at the Los Alamos Scientific Laboratory (LASL) is to demonstrate the technology required to build small, high-energy proton linear accelerators (linacs) for use in generating negative pions for cancer therapy at larger medical centers. Because of the need for a flezible, easy-to-use controller to acquire data from and to control experiments on prototype structures, we developed the microprocessor chassis (MPC) using the basic concept of the FNAL system.

Many of the basic modules for the MPC are commercially available.* Additional modules have been designed and are described in this report. Although in this report we describe a system-type controller, the modules can be used in smaller configurations for dedicated applications and/or single installations."

OMNIBYTE Corp., 245 W. Roosevelt Rd., West Chicago, Illinois. 


\section{THE MPC}

The MPC (Fig. 1a) is $216 \mathrm{~mm}$ high, $482 \mathrm{~mm}$ wide, and $508 \mathrm{~mm}$ deep. The main operating part of the MPC is the microprocessor crate, a 16-slot board cage with a printed circuit (PC) board backplane. The microprocessor, memory, and various interface boards plug into this crate. The basic arrangement of the crate is described in Ref. 1 . The position of the crate allows access to the boards from behind the MPC. The power supplies, mounted beside and in front of the crate, connect to a terminal strip that terminates the power supply wires from the backplane.

Figure 1b shows a locking toggle switch on the lower left front panel for on/off control of the power supplies. The ac-power indicator lights are directly above the switch. There is a reset button on the upper left-hand corner of the front panel.

At the top of the front panel is a four-digit hexadecimal (hex) light-emitting diode (LED) display. All front pane! indicators and switches are accessed through the controller software. Below the hex display are eight discrete LEDs and eight toggle switches. The software controls the LEDs, which operate independently from the toggle switches. The toggle switches set data lines high or low. The data lines also are accessed through the controller software. The LEDs and toggle switches are mounted on one binary switch/LED board. Below the toggle switches is a four-digit hex thumbwheel switch, which is used to enter hex-encoded data into the controller. The hex switch and hex display can be used with binary-coded decimal (BCD) instead of hex format for operators who are more familiar with the BCD format.

The final interface is the push-button/LED board. The toggle switches function like sense switches; they cannot be reset by the microprocessor unit (MPU). The push-button/LED interface board was designed to be used in two different modes. One is the set-reset mode, in which a single push of the button sets a condition. The MPU responds to and resets this condition, whether or not the button has been.released. A second mode is the duration-ofpush mode. Here a push of the button sets the condition, but the condition cannot be reset until the button is released. The first option generally is used for tasks that run independently to completion. The latter option is used to control the duration of a task.
For example, pushing a button opens a valve continuously until a certain pressure is read on a meter. When the button is released, the valve remains in its position, and the pressure remains constant. The sofiware controls the LEDs on the board, regardless of the status of the switches.

Note that the front panel has no labels for the switches and LEDs. This is because the functions of these devices ure dynamic; that is, the functions can be redefined by whatever task is running at that time. We describe this redefining in more detail in Sec. III. For dedicated applications, switch and LED labels probably would be used.

Long cables attach to the 25-pin D connectors on the back panel (Fig. 1c). The D connectors attach to the PIA boards with short lengths of ribbon cable, which can be easily connected to and disconnected from the PLA board. The power supply test points on the lower left-hand side of the rear panel allow the supply voltages to be monitored without removing the MPC from a rack. A fuse, an ac-power-present light, and a power connector that is an integral part of an electromagnetic interference (EMI) filter are mounted on the rear panel. Shown on the upper right-hand side of the rear panel are three Lemo (Kloc) sockets, one of which is an output port whose state is controlled by the software. The other two sockets can be used as inputs to interrupt the MPU. One, a maskable interrupt line, responds to a .positive or negative edge, depending on how it is programmed. The other, a nonmaskable interrupt line, is always active and responds to a high-to-low transition.

The front panel boards are interfaced to the MPU using the standard PIA board. Because the cables can be disconnected at either end, the front panel boards can be removed easily for maintenance.

The hex display board receives its data from the MPU via a dedicated PIA port whose 16 data lines are programmed as outputs. The 16 lines are divided into groups of 4 and each group connects to one of 4 TIL 311 displays, which have their own decoding logic. The hex display board also has two inputs that can be programmed to interrupt the MPU on either positive or negative transitions of signals driving these inputs. These inputs are connected to the CB1 and CA1 lines. One other line, CA2, can output pulses to an external device under software control. The CA2 and CAi lines are connected to the rearpanel Lemo sackets with RG-174 cables. 


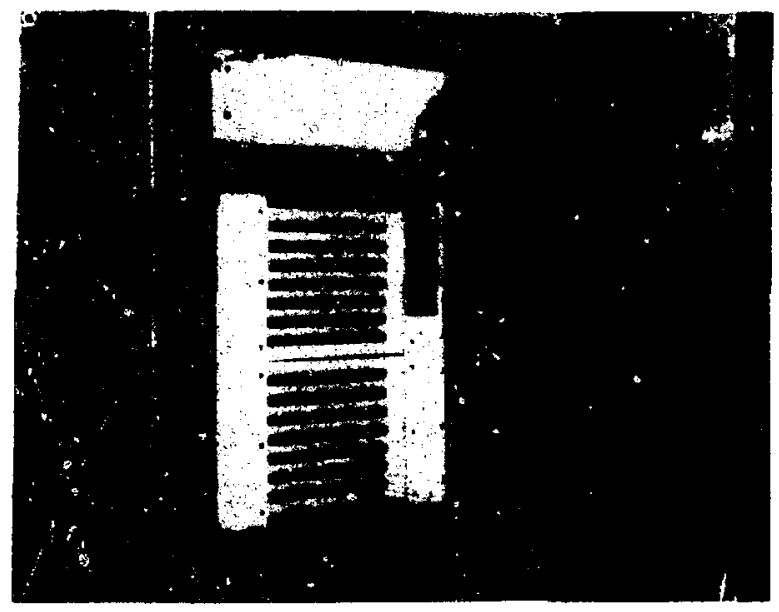

Fig. 1 .

The MPC. (a) top, (b) front, (c) rear.
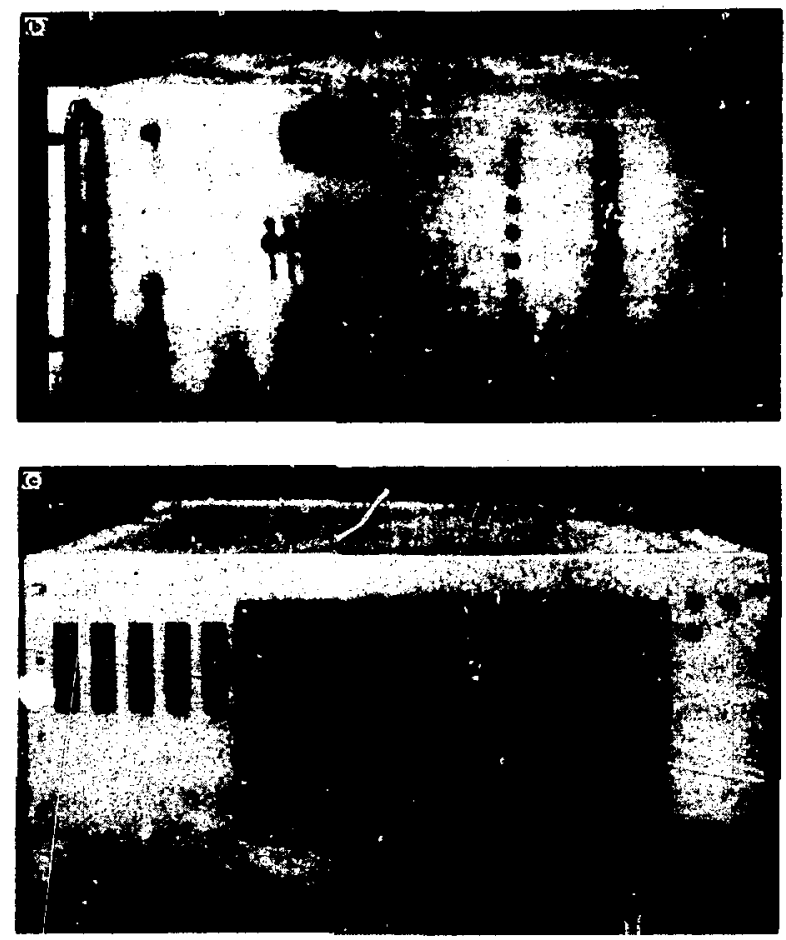

transmits an interrupt to the MPU. By another method, the MPU polls the data lines to check whether any of them are set. In either case, the MPU, through the PIA, can asynchronously reset the flip-flop, whether or not the button is still depressed. In the duration-of-push mode, the initialization of the board gates the switch status instead of the outputi of the flip-flops onto the data lines. Again, the pushing of a switch may or may not cause the MPU to be interrupted, but the computer cannot reset the action. Because this pushbutton/LED board has not been reported before, a schematic and a software listing of a diagnostic program for it are provided in Appendix $A$.

\section{INTERACTIVE FRONT PANEL AND TELETYPE MONITOR}

We developed a versatile monitor program that can perform tasks in a loop and can service an ASCII-encoded keyboard terminal (KBT).

The basic loop in all systems performs two functions. The first function checks whether a button has been pushed; if so, the task assigned to that push button is executed. The second function is executed 
if buttons 6 and 7 are pushed within $50 \mathrm{~ms}$ of each other; when this occurs, the value entered on the toggle switches is stored in the memory addrese specified on the her switch. To accomplish the first function, a vector, or two random access memory (RAM) locations, are defined for each push button. These vectors contain the address of the task currently assigned to a button. (Appendix $B$ lists the button numbers and associated RAM locations that contain the vectors.) When a button is pushed, program control jumps to the subroutine pointed to by that button's vector, executes the subroutine, and retums to the monitor loop. A button task is changed simply by puiting a different subroutine address in the proper vector location. Either software reassignment of the vector or use of a vector assigiment feature of the front panel can change the task. To use the latter feature, one must dial the new task address on the hex switch, raise the toggle switch that corresponds to the button to be reassigned, and push button 8 , the lower push button. Using this feature assigns the address on the hex switch to the button designated by the toggle switch. Button 8 is assigned this feature at initialization, although its own vector cannot be reassigned by using the feature. However, a new vector can be assigned to button 8 by the software.

In addition to the monitor's two basic functions, other functions can be added to every loop while it is checking for a pushed button or after a pushed button has been detected, but before the associated task is executed. Figure 2 shows where these functions can be added. The loop tasks have RAM vectors, which are similar to the push-button RAM vectors (Appendix B). To add functions to the loop, store the address of the subroutine that provides the function in the proper vector.

Two resident functions that can be added to any loop are a function that displays memory in the hex display and a function that provides an activity monitor. The first. function displays in the hex display the data in the memory address specified by the hex switch and also the data in the next sequential memory location. The lights above the toggle switches are the activity moritor. Their rate of movement reflects the activity of the system. Between each move of the lights, the monitor loop is executed 1024 times.

The standard front panel monitor software is used in each MPC of the PIGMI control system. The

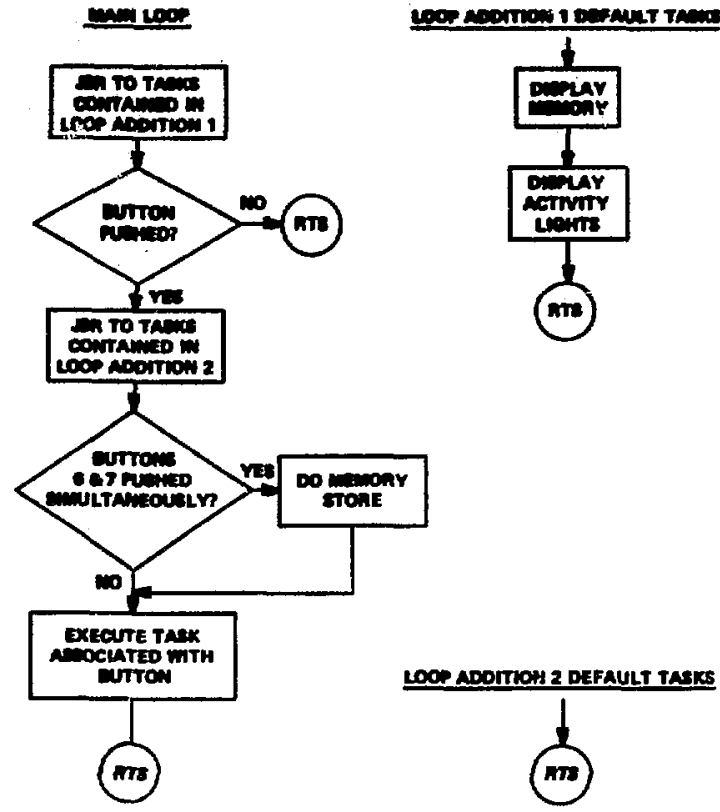

Fig. 2.

Front panel monitor flow chart.

software is expanded to perform all necessary functions in a particular system. For example, the MPU that monitors the consule has features in the main loop that service the touch panel, character scope, trackball, keypad, 611 storage scope, NOVA-MPC communications, etc.

Listing 1 in Appendix $\mathrm{C}$ is an example of the basic front panel monitor software. The desired features are added to the basic loop lines 29 to 35 in the subroutine SUB (lines $36-39$ ). The routine to service a pushed button is in lines 65-96. This basic software also includes routines to

- initialize button and loop vectors (lines 99-118),

- initialize the front panel boards (lines 119-145),

- reset the push buttons (lines 146-150),

- displey data at the memory address dialed in the hex switch (lines 158-161),

- use button 8 to reassign the button functions (lines 164-177),

- display activity lights (lines 180-191), and

- delay microseconds or milliseconds (lines 196215).

System access to a KBT is sometimes desirable, especially during the developmental stages. Therefore, we made the KBT monitor (KBTMON) used in the software development system coresident 
with the MPC system software. To merge the two monitors, four guidelines were considered.

(1) Usually, the panel monitor loop should not be disabled for more than a few milliseconds.

(2) The KBTMON software will be standard in all systems.

(3) The tape load function of KBTMON should not be degraded; that is, tapes should dependably load at 1200 baud.

(4) The merger should be done in a straightforward manner that requires minimal software communication between the monitors.

A straightforward merger provides that the pushburton monitor (PBMON) be serviced on each entry into an input/output (I/O) routine of KBTMON. With a microprocessor clock rate of $1 \mathrm{MHz}$ the delay between PBMON loops is $75 \mu \mathrm{S}$ when no entry is made from the KBT, $100 \mu 8$ when an entry is processed, and variable when a memory zero or transfer function is executed.

Through this merger, PBMON is intact and complitely independent of KBTMON, and KBTMON transfers control to PBMON instead of wasting time while waiting for $\mathrm{I} / \mathrm{O}$ timing. For example, a KBT operating at 300 baud transmits a character every $3.3 \mathrm{~m}$ at its fastest rate. The input loop is $100 \mu \mathrm{s}$ long. Instead of waiting in a loop for the next input, KBTMON transfers control to PBMON, which usually returns control to KBTMON in less than 200 $\mu s$. When operating at 300 baud, the asynchronous communications interface adapter (ACIA) board can be neglected for $\sim 6.6 \mathrm{~ms}$ without losing KBT input data. Losing data, which rarely happens, is not criticai because the loss is signaled to the operator, who then re-enters the data. A complete listing and documentation of the adapted KBTMON is given in Appendix D.

In this scheme, KBTMON must know the initialize and loop vectore of PBMON (Appendix B). Because PBMON is independent of KBTMON, one can use PBMON, without modification, with any type of KBT monitor. Only minor modification of the KBT monitor is required.

\section{ACKNOWLEDGMENTS}

We thank M. F. Shea and R. W. Goodwin of FNAL for transferring the microprocessor technology to LASL, K. R. Crandall and G. R. Swain of LASL for implementing the cross assembler, and V. A. Martinez, who did the technician and drafting work.

\section{REFERENCES}

1. R. W. Goodwin, R. F. Kocanda, and M. F. Shea, "A Method for Implementing Microprocessor Controlled Systems," IEEE Trans. Nucl. Sci. NS-23, No. 1, 297-300 (1976).

2. D. J. Liska, J. N. Levitt, J. K. Halbig, and L. B. Dauelsberg, "A Clinical Servo System for Shifting the Range of Pion Beams," IEEE Trans. Nucl. Sci. NS-24, No. 3, 1046-1047 (1977).

\section{APPENDIX A}

\section{PUSH-BUTTON LED BOARD SCHEMATIC AND SOFTWARE LISTING}

See Fig. A-1 inside pocket at the back for schematic. 


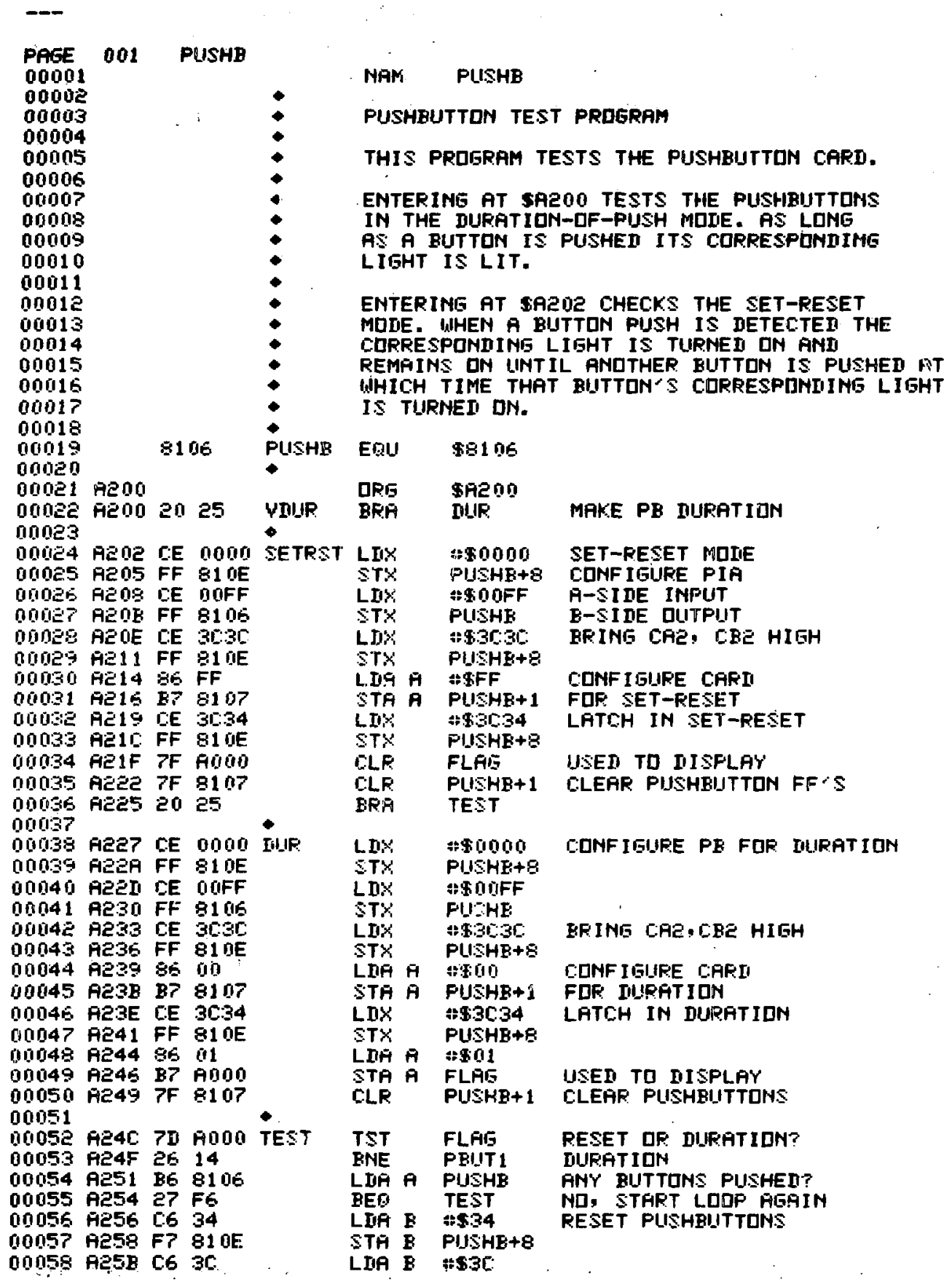




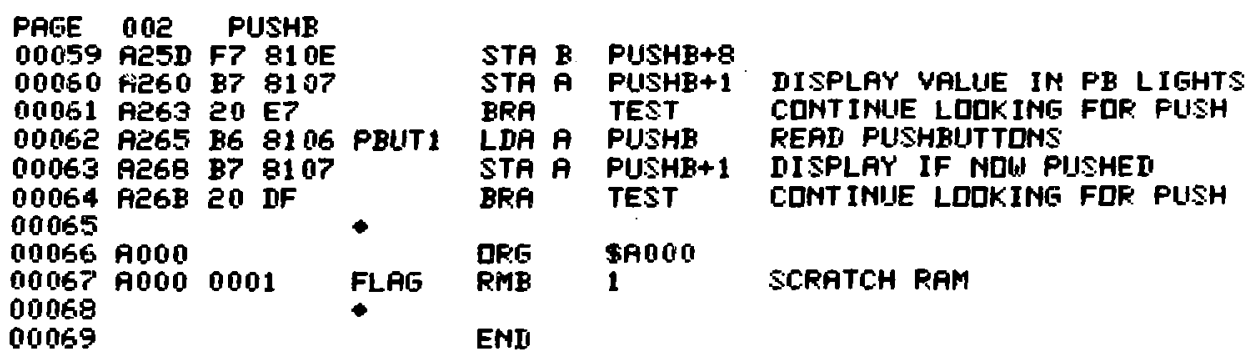

TOTAL ERRORS 00000

\section{APPENDIX B}

\section{VECTORS}

TABLE B-1

\section{SYSTEM VECTORS}

\section{Vector}

Button-1 task address Button-2 task address Button-3 task address Button- 4 task address Button-5 task address Button- 6 task address Button- 7 task address Button- 8 task address
PBMON Location (hexadecimal)

A1E0

A1E2

A1E4

A1E6

A1E8

A1EA

A1EC

A1EE

- On restart, these button vectors default to the tasks described by vectors \$F003-\$F017 under PBMON vectors.

TABLE B-II

\section{LOOP VECTORS}

\section{PBimON Loop Addition Vectors" (hexadecimal) \\ A1F0 First loop addition A1F2 Second loop addition}

On restart, these vectors default to the tasks described in Fig. 2 (in text). 


\section{PBMON VECTORS}

\begin{tabular}{clll} 
Location & Name & & \multicolumn{1}{c}{ Description } \\
\cline { 1 - 2 } F000 & VPLOOP & Goes to beginning of monitor locp \\
F003 & VBUT1 & Goes to button-1 task \\
F006 & VBUT2 & Goes to button-2 task \\
F009 & VBYT3 & Goes to button-3 task \\
F00C & VBUT4 & Goes to button-4 task \\
F00F & VBUT5 & Goes to button-5 task \\
F012 & VBUT6 & Goes to button-6 task \\
F015 & VBUT7 & Goes to button-7 task \\
F018 & VINALL & Initializes all devices, vectors used in PBMON \\
F01B & VIPANL & Initializes only front panel PIAs \\
F01E & VRSX11 & Produces activity lights \\
F021 & VMILLI & Delay (X) milliseconds \\
F024 & VMICRO & Delay (X) microseconds
\end{tabular}

TABLE B-IV

KBTMON VECTORS

Location (hexadecimal)

FCO0

FC06

FC09

FCOC

FCOF

FC12

FC15

FC18

FC1B

FC1E

FC21

FC24

$\mathrm{FC} 27$

FC2A

Name

Description

VENTR Initializes ACIA, RAM, goes to TTY control loop

VINHEX Checks whether character is valid hex character

VBADDR Inputs 4-digit address

VOTCH Outputs 1 charecter

VOT2H Outputs 2 characters

VOT4HS

Outputs 4 characters and I space

VOT2HS

VOTS

Outputs 2 hex characters and 1 space

VPDAT1

Outputs 1 space

VPDATA

Prints data string

VPCRLF

Prints carriage return, line feed, data string

VPSTK

VBYTE

Prints carriage return, line feed

Prints stack on interupt

VBINH

Inputs 1 byte

No action

VINCH Inputs 1 character 


\section{APPENDIX C \\ LISTING 1 \\ LASL Identification No. LP-1067 (TTYMON)}

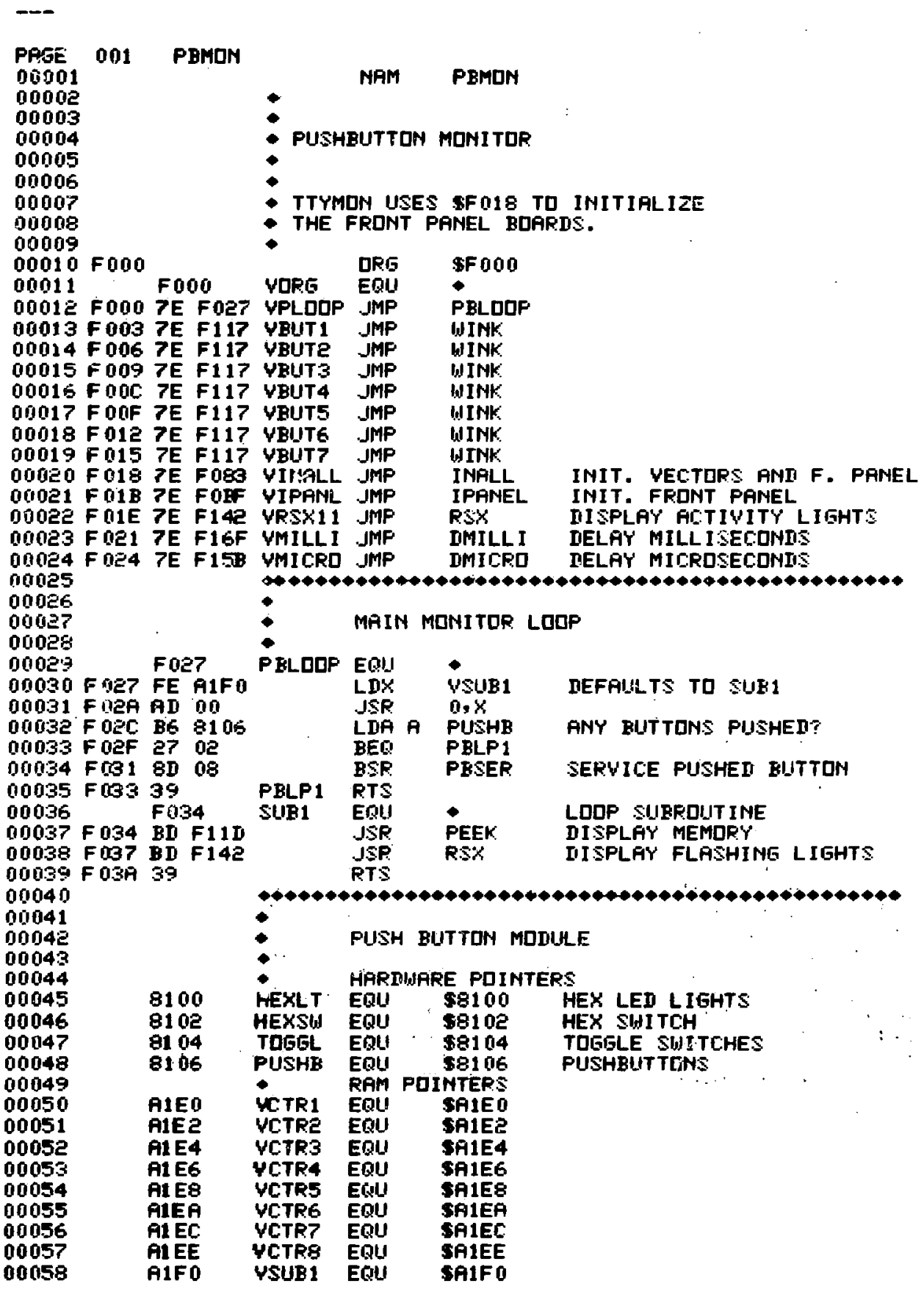




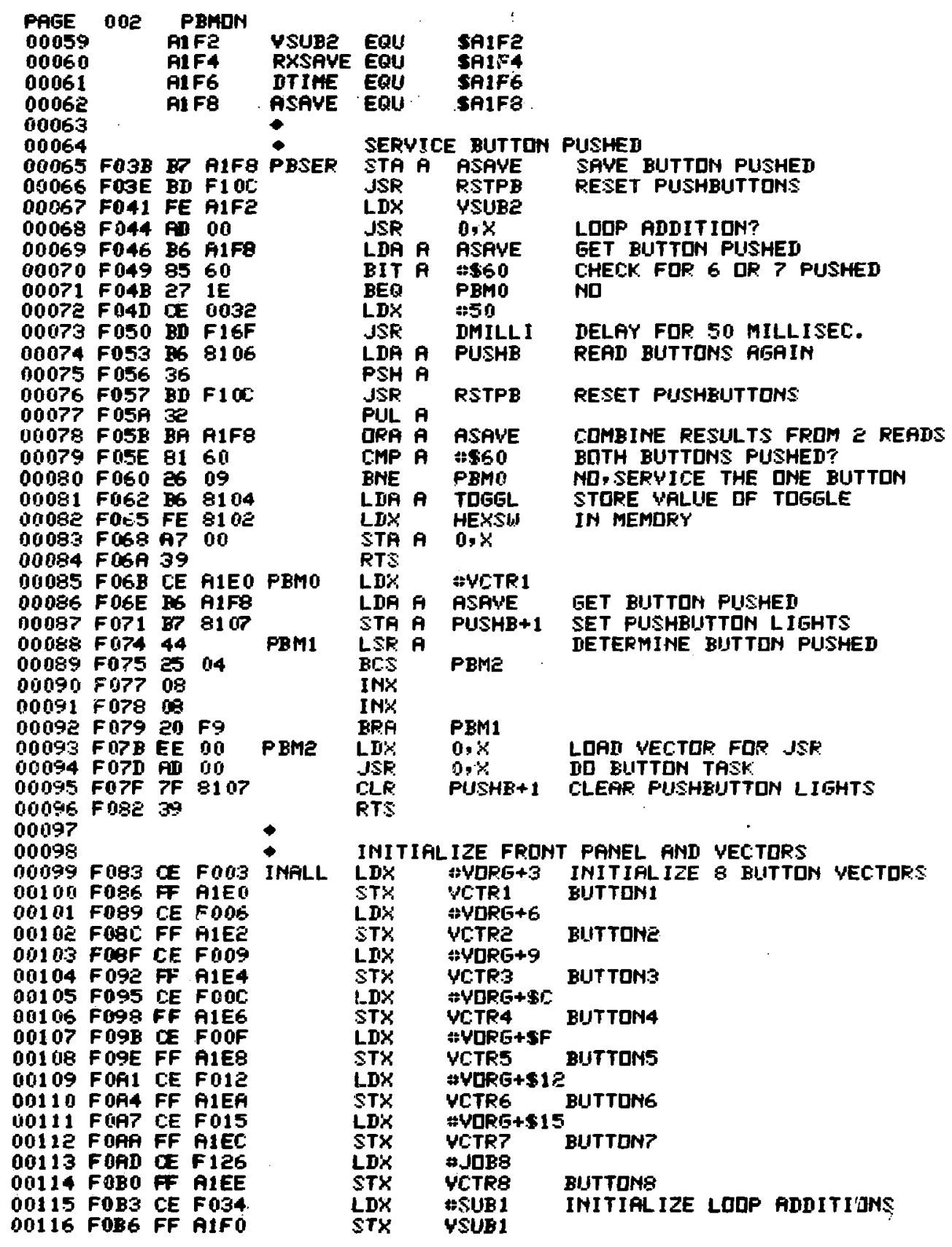




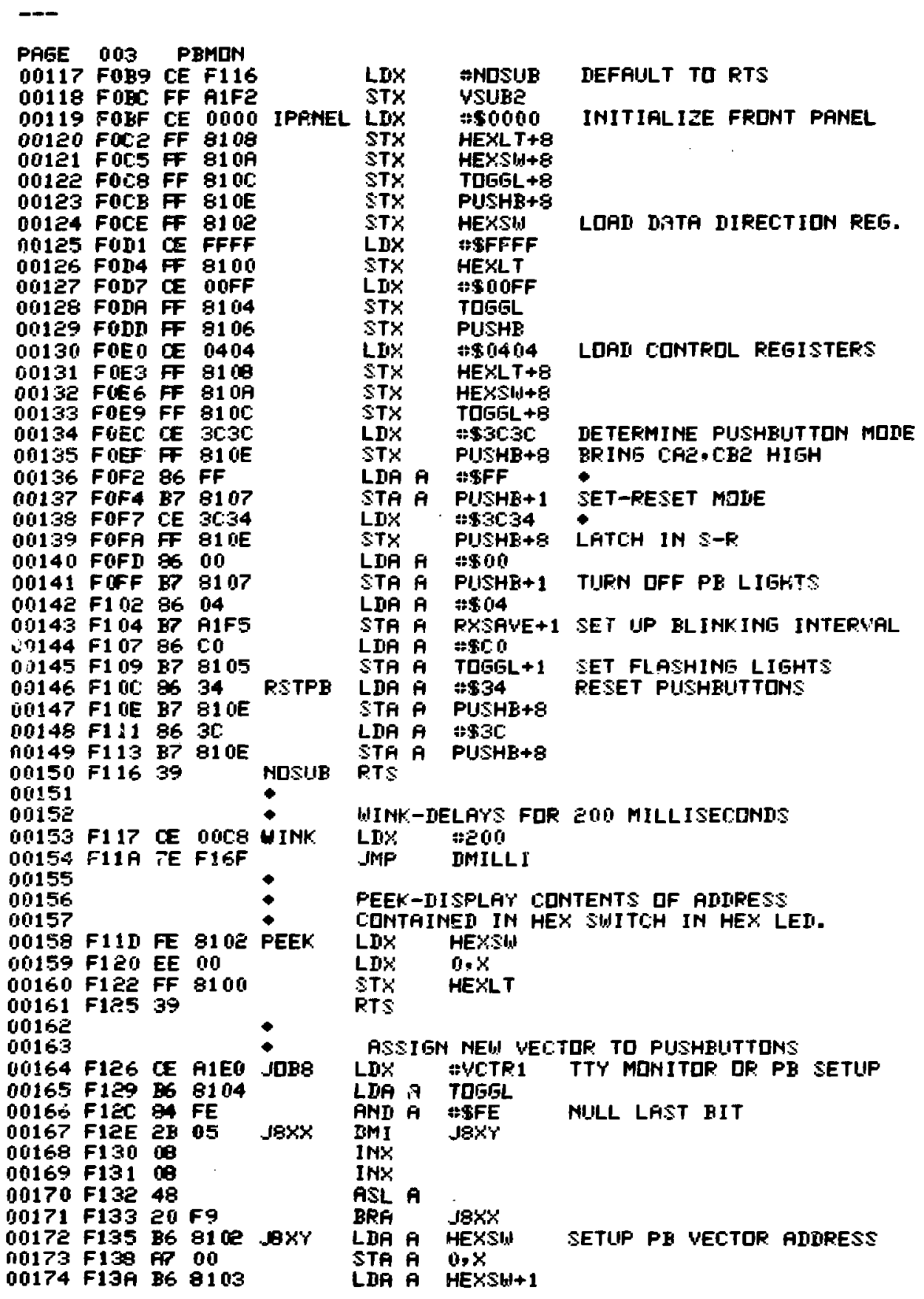




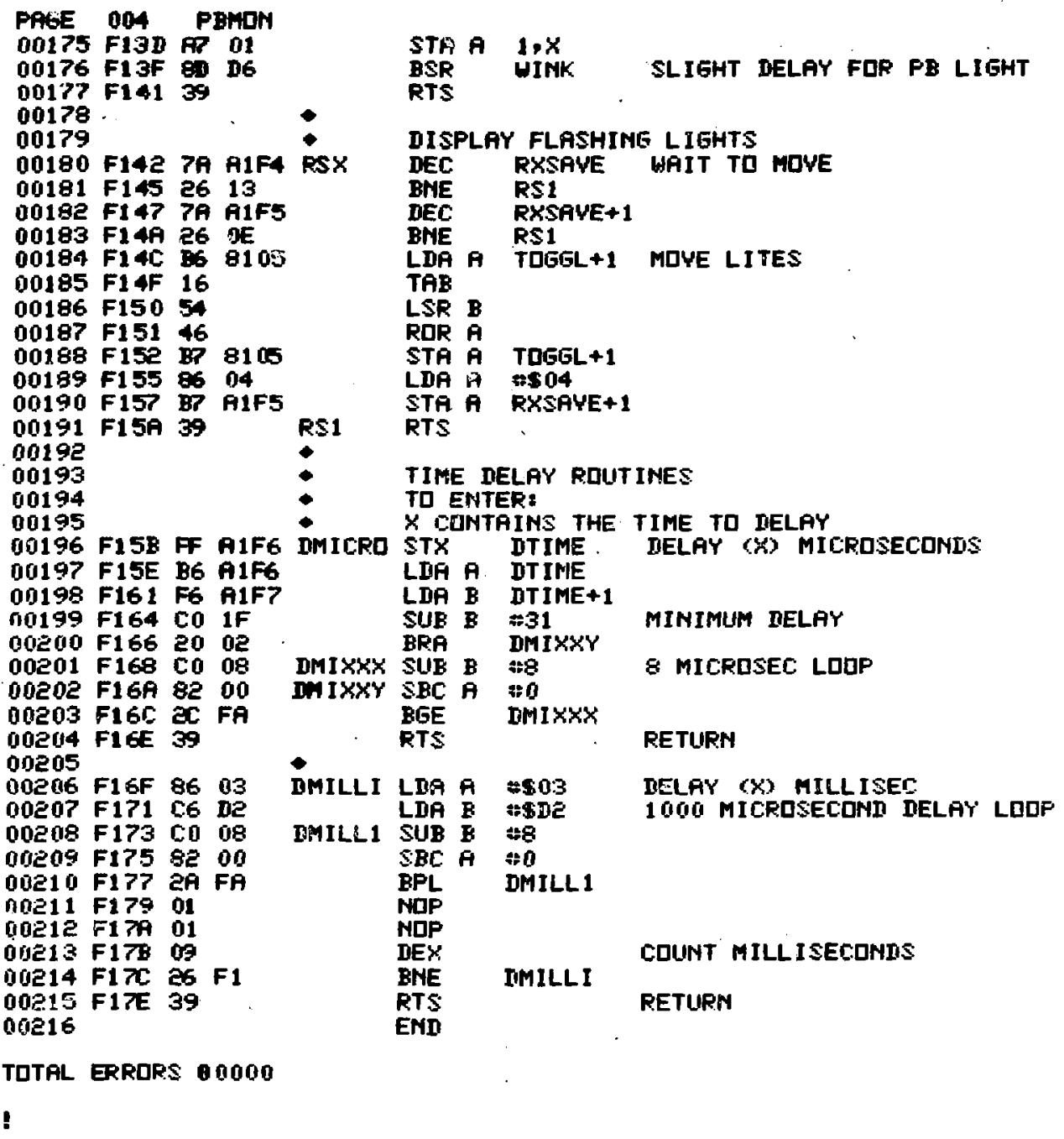

\section{APPENDIX D}

KBTMON AND LISTING

\section{GENERAL}

The keyboard terminal monitor (KBTMON) program is written in MC6800 microprocessor assembly language for use with any American Standard Code for Information Interchange
(ASCII)-encoded KBT. The DUMP and LOAD functions are written for Texas Instruments (TI) 733 or ASR33 with automatic tape-control options. The program resides at locations \$FCOO thurough \$FFFF (a \$ indicates a hex No.) in read only memory (ROM) and uses RAM (random access memory) 
\$A100 through \$A1FF locations for temporary storage and for the MPU stack. The ACIA board must be in slot 5 .

KBTMON is entered by a restart or jump to the START vector at $\$ F C 00$. At entry, KBTMON responds with a carriage return, line feed, and asterisk $\left.{ }^{*}\right)$; then it goes to the KBTMON control routine, which continuously monitors the ACIA receive data register for input from the KBT. When a valid input is received, the control routines branch to a task specified by the input character. These characters are valid inputs.

M-MEMGRY-examines and/or changes memory location.

G-GO-transfers control to another routine.

D-DUMP-dumps memory onto KBT or TI output.

L-LOAD-loads tape.

P-PUNCH-punches memory onto punch paper tape or cassette.

$\mathrm{C}-\mathrm{COMPAR}$-coinpares two regions in memory.

Z-ZEROM-sets memory locations to zero.

$\mathrm{X}$-MOVEM-moves memory.

Inputting one of these letters executes a subroutine. (The name of the subroutine follows the letter above.) Control is returned to the KBTMON control entry point after successful execution of a routine.

\section{MAIN SUBROUTTNES}

The following is a description of each subroutine with instructions for executing that routine. Each subroutine can be used by other programs. Programs that use the $L$ or $M$ options must do a stack save, or STS $\$ A 195$, and a save of the address to which control is returned if an input error is encountered. This address should be stored with its high-order byte in \$A19C and low-order byte in \$A19D. The default address is that of KBTMON control.

\section{A. MEMORY $-\mathbf{M}$}

A memory location can be examined and/or changed by typing $M$ and the four hex digits representing the address (ADDR).

To execute MEMORY,

(1) Type M

(2) System responds with a space. Type the 4digit hex address.
(3) System responds with a carriage return and line feed, prints ADDR and its contents, and waits.

(4) (a) To examine the next memory location, type carriage return. System will respond as in step (3) at the next memory location, ADDR+1.

(b) To change the memory, type the hex characters. (Invalid characters cause the system to respond as in step (3) at ADDR+1.)

(c) To return to KBTMON control, type any nonhex character except a carriage return.

\section{B. GO-G}

GO transfers control to another program or subroucine. The program counter is loaded with the address in locations \$A190-\$A191 (XBRNH). Control is retumed to KBTMON when an unpaired RTS is encountered.

To execute GO,

(1) Type G

(2) System prints ADDR ? where ADDR is the address to which control will be transferred.

(3) Do one of the following.

(a) To transfer to address specified by step (2), type carriage return.

(b) To change the transfer address, enter a new transfer address.

(c) To exit before any transfer occurs, type any nonhex character except a carriage return.

\section{DUMP-D}

Specified memory contents are dumped on the keyboard in even blocks with the line format (beginning address followed by $16_{10}$ bytes of memory).

To execute DUMP,

(1) Type D

(2) System prints ADDR1 ADDR2 ? where ADDR1 is the beginning memory address and ADDR2 is the endinf address of the segment to be dumped.

(3) Do one of the following.

(a) To dump memory specified by step (2), type carriage return. 
(b) To specify a new memory segment, type a new beginning address. System risponds with ? Type a new ending address.

(c) To exit the DUMP mode before any action is taken, type any nonhex character except a carriage retum.

(4) To abort the routine while memory is being dumped, type $S$ to retum control to the loop and wait for a character to be input.

\section{LOAD-L}

The contents of a cassette or paper tape are loaded into memory at either the address on the tape or an address specified from the keyboard. A checksum is calculated and compared to the one on the tape. If a checksum discrepancy occurs, ? is printed, the tape is turned off, and control returns to KBTMON.

To execute LOAD,

(1) Position the tape at the beginning of the information to be loaded. If using a TI terminal, turn on the automatic, or remote, device control (ADC).

(2) Type L

(3) System responds with ?

(4) Do either (a) or (b).

(a) To load the tape into memory at the address that is on tape, hit the carriage return.

(b) To load the tape into memory at an address other than that on tape, enter the address that is on the tape. System responds with ?

Enter the new address.

\section{E. PUNCH-P}

The contents of the memory between specified addresses are dumped on cassette or punch paper tape in Motorola's standard format shown in Fig. D-1.

To execte PUNCH,

(1) Tum ADC on if at TI terminal.

(2) Type $P$

(3) System prints memory ADDR1 ADDR2 ? where ADDR1 is the beginning memory ad- dress and ADDR2 is the ending memory address of the segment to be punched.

(4) Do one of the following.

(a) To punch the memory specified by step (3), hit carsiage seturn.

(b) To specify a new segment of memory, type a new beginning address.

System responds with ?

Type a new ending address.

(c) To exit the punch mode before any action is taken, type any nonhex character except a carriage return.

(5) To abort the process while memory is being punched, type S. KBTMON returns to the control loop and waits for a character to be input.

\section{F. COMPAR-C}

Memory segment $A$ is compared with memory segment B. Any differences are signaled by an error message of the form M ERR ADDF XX YY, where ADDR is the memory $\mathrm{B}$ address, $\mathrm{XX}$ is the memory $B$ contents, and $Y Y$ is the memory $A$ contents.

To execute COMPAR,

(1) Type C

(2) System responds with ADDR1 ADDR2 ADDR3 ? where ADDR1 is the beginning memory $A$ address, ADDR2 is the ending memory $A$ address, and ADDR3 is the beginning memory $B$ address.

(3) Do one of the following.

(a) To compare memory specified by step (2), type carriage return.

(b) To specify new segments of memory, enter a new beginning memory $A$ address. System responds with ?

Enter a new ending memory $A$ addiress. System responds with ?

Enter a new beginning memory $B$ address.

(c) To exit the compare mode before any action is taken, type any nonhex character except a carriage return.

(4) To abort the process while compare errors are being printed, type $S$ to return control to the loop and wait for a character to be input. 


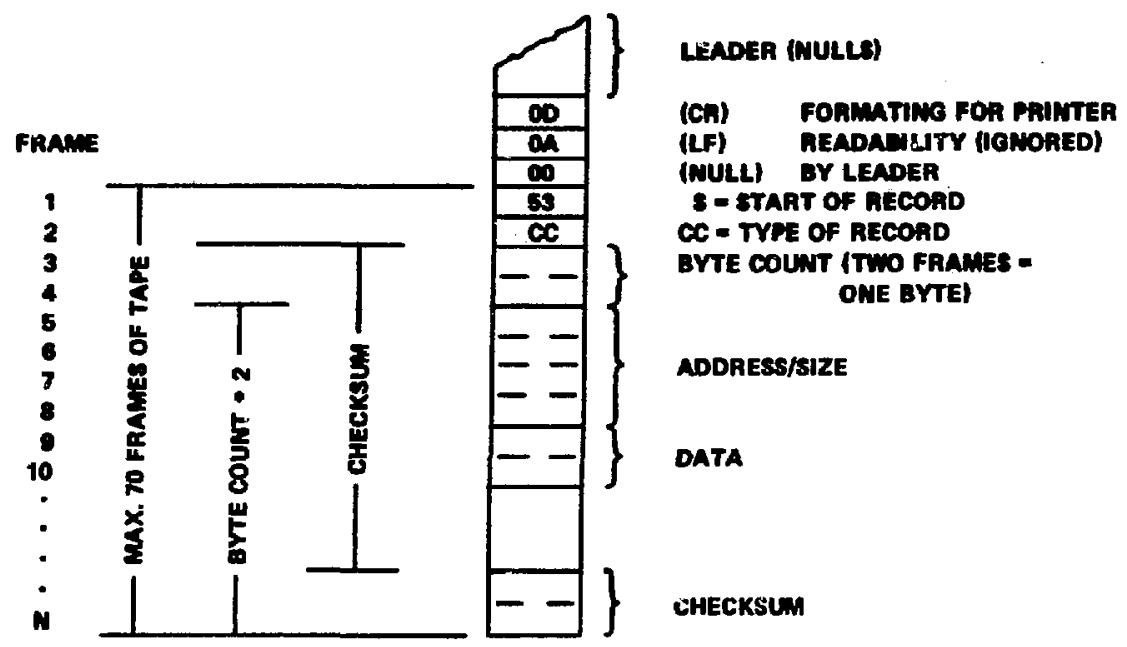

FRAMES 3 THROUGH N AAE HEXADECIMAL DIGITS (IN 7-EIT ASCII) WHICH ARE CONVEATED TO BCD. TWO BCD DIGITS AAE COMBINED TO MAKE ONE \&-BIT BYTE.

THE CHECKSIM IS THE ONE'S COMPLEMENT OF THE SUMMATION OF \&BIT BYTES.

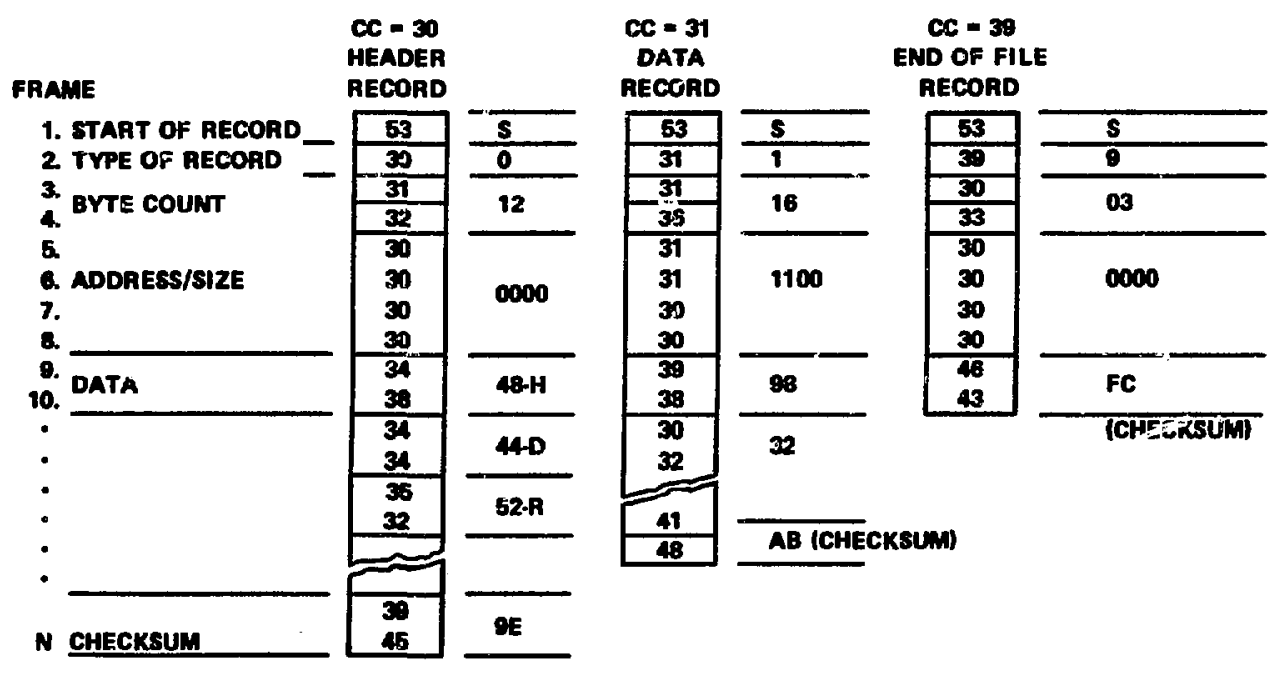

Fig. D-1.

Motorola standard tape format. (This figure is reproduced by permission from Motorola Engineering Note 100.)

\section{G. ZEROM-Z}

All specified memory locations are set to zero.

To execute ZEROM,

(1) Type Z
(2) System responds with ADDR1 ADDR2 ? where ADDR1 is the beginning memory address to zero and ADDR2 is the ending memory address to zero. 
(3) Do one of the following.

(a) To set the memory specified in step (2) to. zero, type carriage return.

(b) To specify a new memory to be set to zero, type a new beginning memory address to zero.

System responds with ?

Type a new ending memory address to zero.

(c) To exit the zero mode before any action is taken, type any nonhex character except a carriage return.

\section{H. MOVEM-X}

Memory contents are moved from one iocation in memory to another specified location. The contents of the newly filied memory are compared to the original memory; any unsuccessful move is signaled by an error message of the form $M$ ERR AD DR XX YY, where $M$ is the memory, ADDR is the address at which the error occurs, $\mathrm{XX}$ is the incorrect memory contents, and $Y Y$ is the original memory contents.

To execute MOVEM,

(1) Type X

(2) System responds with ADDR1 ADDR2 ADDR3 ? where ADDR1 is the beginning memory address to transfer, ADDR2 is the ending memory address to transfer, and $\mathrm{AD}$ DR3 is the beginning memory address to which the move is made.

(3) Do one of the following.

(a) To make the transfer specified by step (2), type carriage return.

(b) To specify a new transfer, type a new beginning transfer memory address.

System responds with?

Type a new ending transfer memory address.

System responds with ?

Type a new beginning address to which to transfer.

(c) To exit the transfer mode before any action is taken, type any nonhex character except a carriage return.

(4) To abort the process while compare errors are being printed, type $S$ to return control to the loop and wait, for a character to be input.

\section{I/O ROUTINES}

The following I/O routines are available for use in applications programs. They are used by JSR \$XX$\mathrm{XX}$ instruction where $\$ \mathrm{XXXX}$ is the hex address of the routine given after the routine name.

\section{A. Input Rautines}

1. INCH \$FC2A. One 8-bit word is loaded from the ACIA input register into the $A$ accumulator. The character is echoed on the terminal.

2. INHEX \$FC03. An 8-bit word is loaded into the $A$ accumulator from the ACIA input register. The character is echoed and checked whether it is a valid hex character, 0-9, A-F. $\dot{A}$ nonhex character causes a return to the specified address.*

3. BYTE \$FC24. Two valid hex characters are loaded. They are converted to their 4-bit binary representation and then packed in the $A$ register. If either of the input characters is not a valid hex character, a return to the specified address is executed.*

4. BADDR \$FC06. Four characters are loaded from the ACIA. The hex characters are converted to their binary representation and packed in the $X$ register. The high-order address byte is also store $d$ in \$A18A; the low-order address byte is storea in \$A18B. The address is echoed. An encounter of a nonhex character causes program control to return to the specified address.*

\section{B. Output Routines}

1. OUTCH \$FC09. One ASCII character is output. The routine is called with the character to be output in the $A$ register.

2. OUT2H \$FCOC. Two hexadecimal characters are output. The routine is called with the memory address of the location that contains the packed hex characters in the $\mathrm{X}$ register.

"Control is returned to the address specified in locations $\$ \mathrm{~A} 19 \mathrm{C}$ and $\$ A 19 D$. KBTMON defaults the return to KBTMON control on entry into KBTMON from a restart. 
3. OUTS \$FCi5. A space is output.

4. OUT2HS \$FC12. OUT2H and OUTS are combined.

5. OUT4HS \$FCOF. Four hex characters followed by a space are output. The characters to be output are packed in two sequential memory locations; the address of the first is entered in the $X$ register before the call is made.

6. PDATA1 \$FC18. A data string is printed. The $\mathrm{X}$ register contains the beginning memory address of the sequential locations that contain the ASCII character representations to be output. Output is terminated when $\$ 04$ is encountered in a memory location.

7. PDATA SFC1B. PDATA is the same as PDATA1 except a carriage return-line feed is output before the ASCII character string.

8. PCRLF \$FC1E. A carriage return and line feed are output.

\section{OTHIGR FEATURES}

\section{A. Stack}

KBTMON can print the contents of the MPU stack. For example, it can dump the contents of the MPU registers at a perticular point in the software. To execute this, load the secondary SWI vector at \$A1FA with \$FC21 and then insert an SWI instruction in the software where you want to know the contents of the MPU registers. The stack contents and the location of the stack pointer are printed on a single line in the order:

condition code register, $B$ accumulator, A accumulator, $\mathrm{X}$ register high-order byte, $\mathrm{X}$ register low-order byte, program counter high-order byte, program counter low-order byte, and stack pointer high- and low-urder byte.

\section{B. Interruye Vectors}

KBTMON creates secondary IRQ, NMI, and SWI vectors. (These interrupts are defined in the Motorola 6800 Applications and Programming manuals.) The address to which an SWI interrupt is to be transferred is entered in \$A1FA and $\$ \mathrm{~A} 1 \mathrm{FB}$; the vector for IRQ is entered at $\$ A 1 F C$ and $\$ A 1 F D$, and the NMI vector is entered at $\$ A 1 F E$ and $\$ A 1 F F$. 


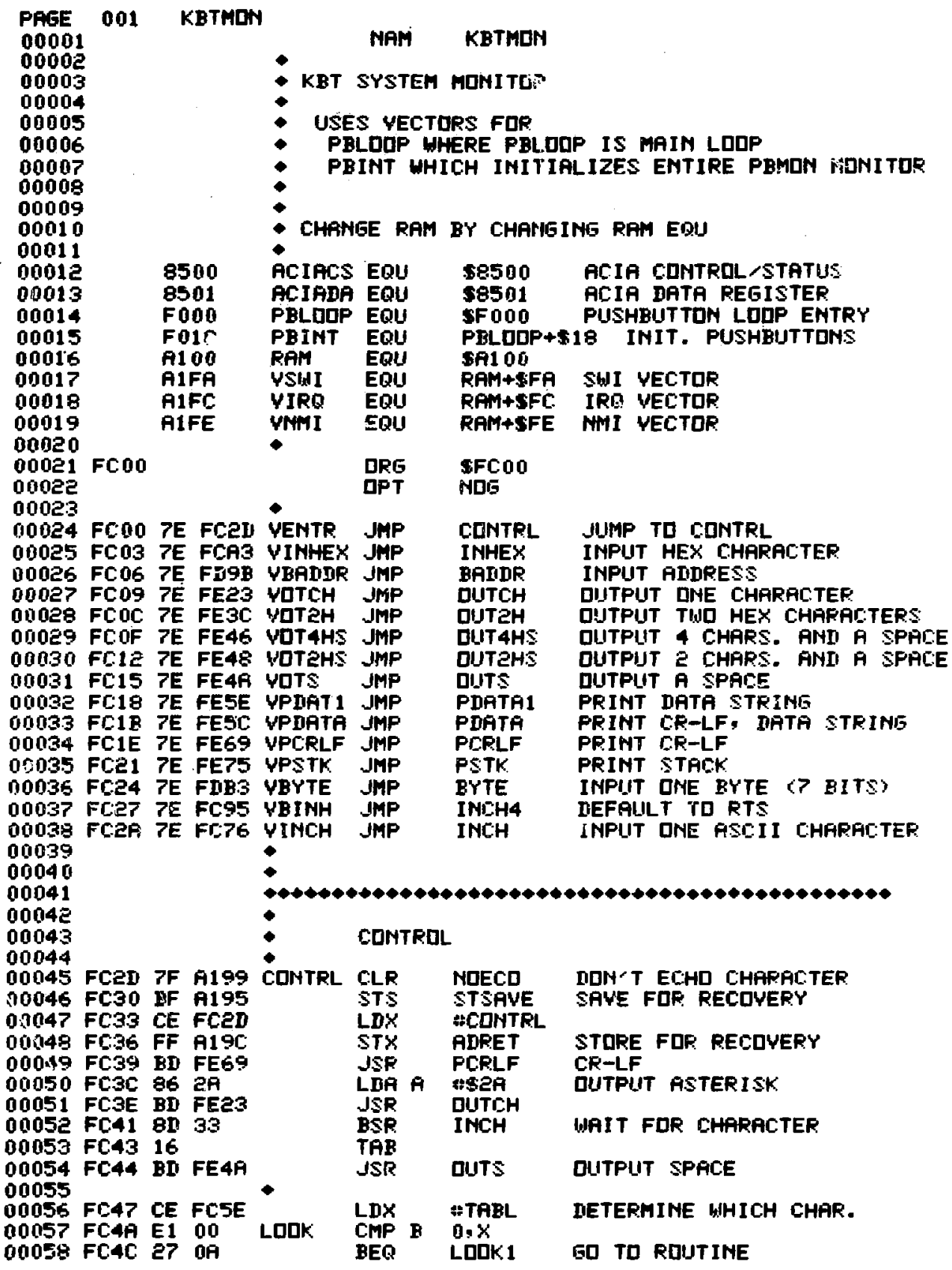




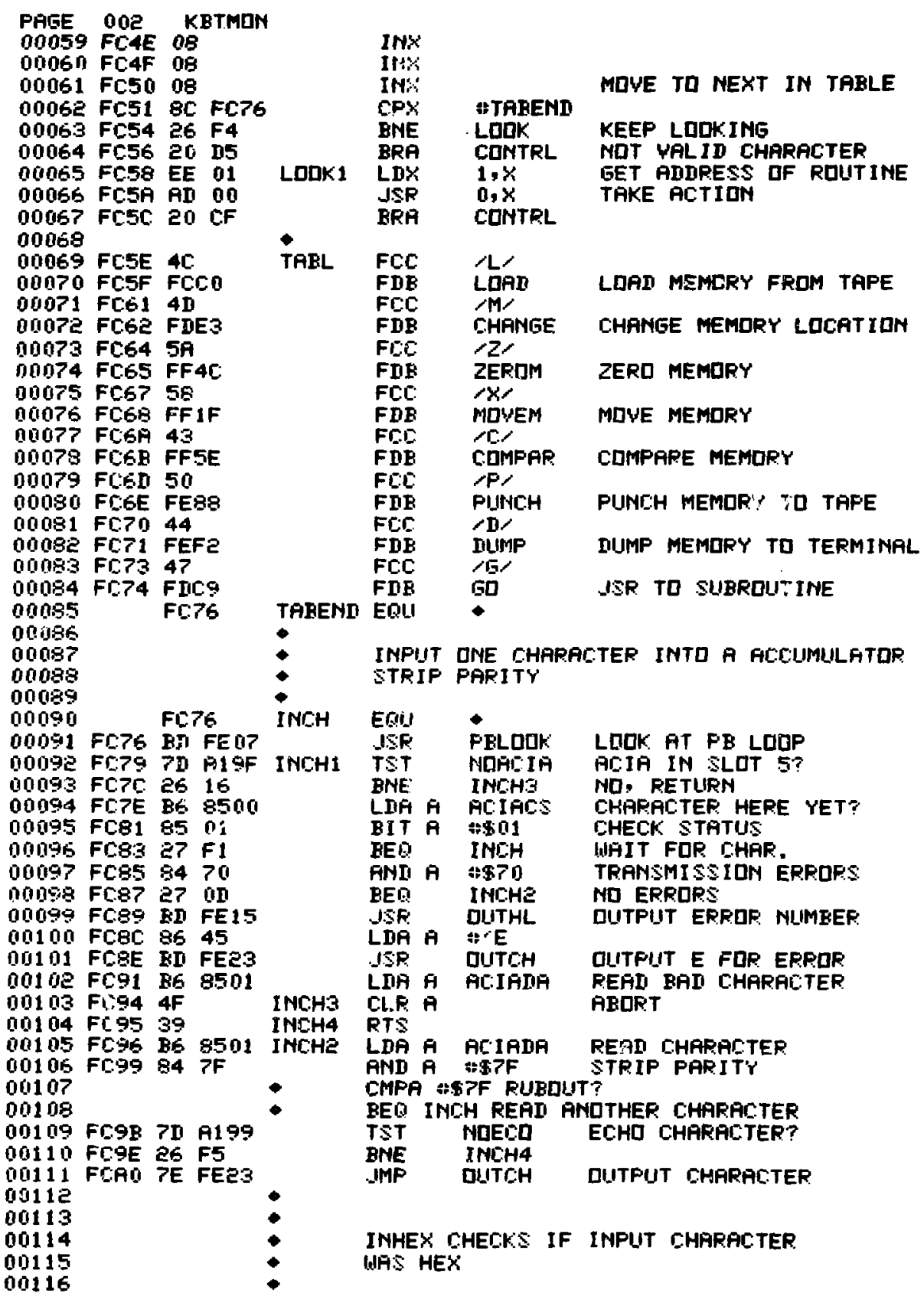




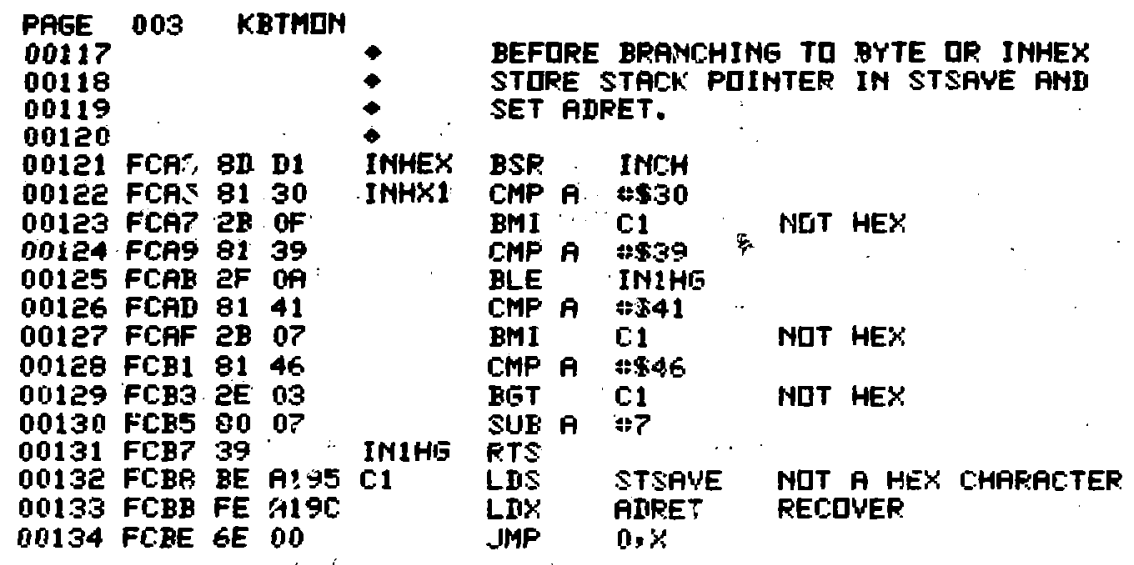




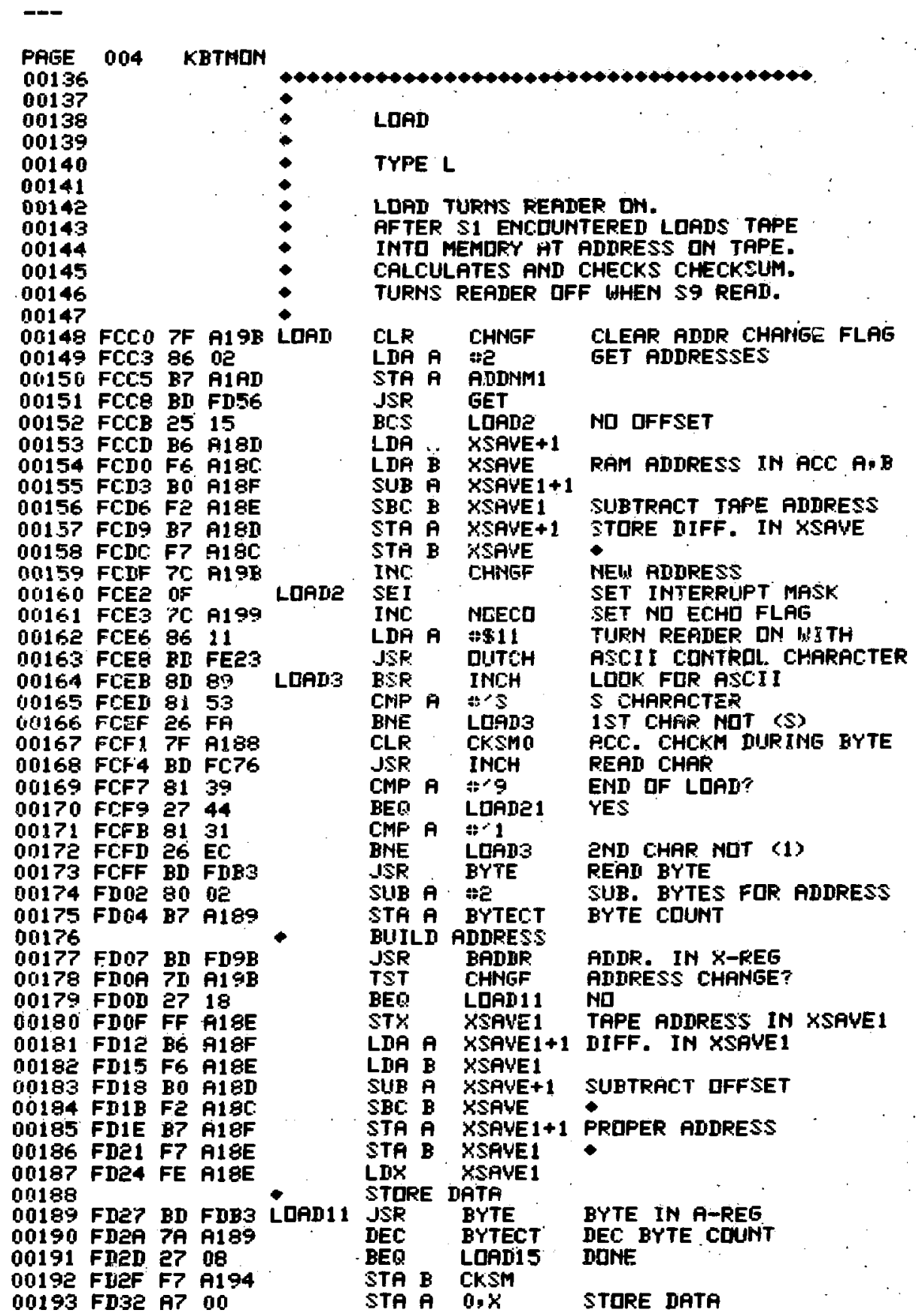




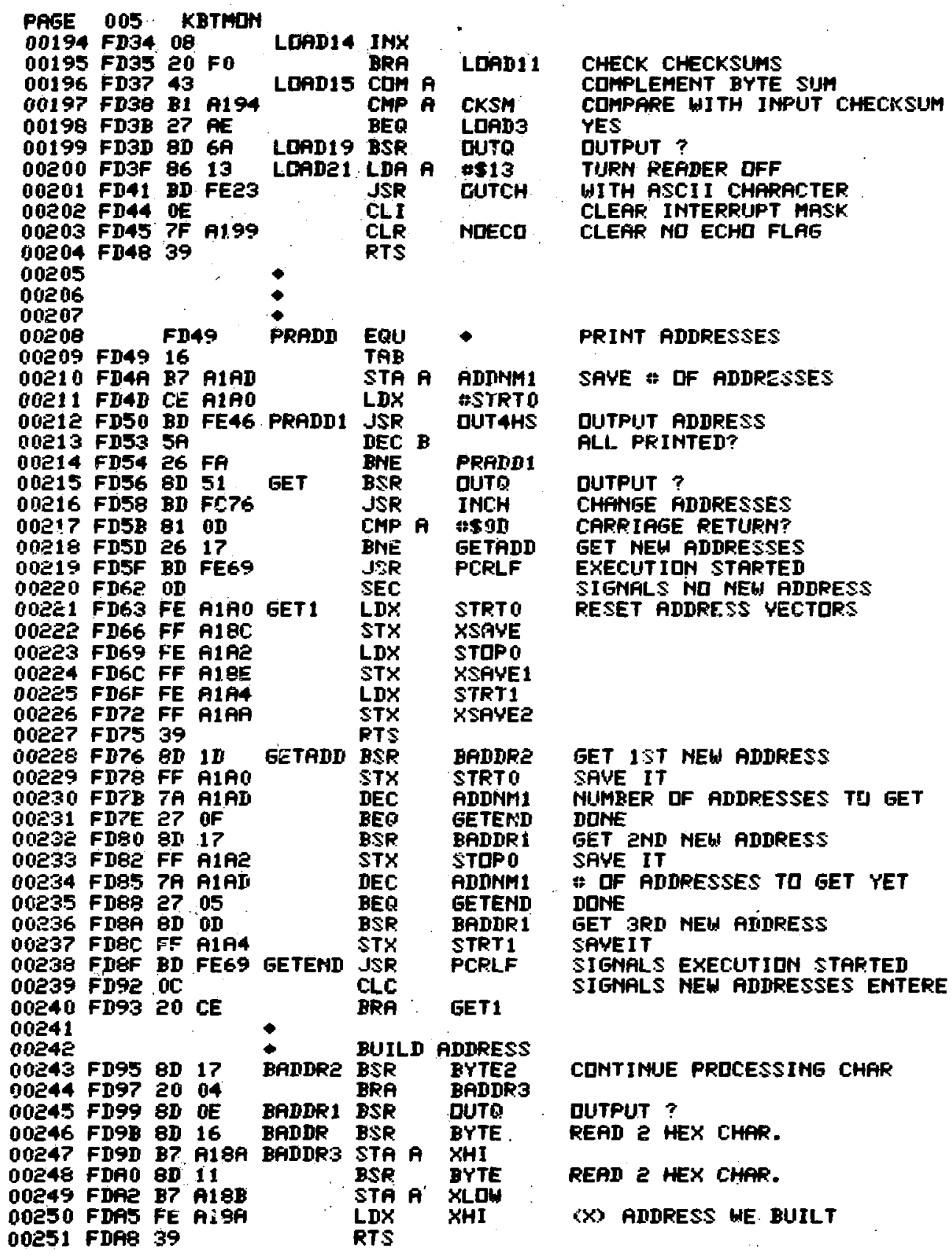




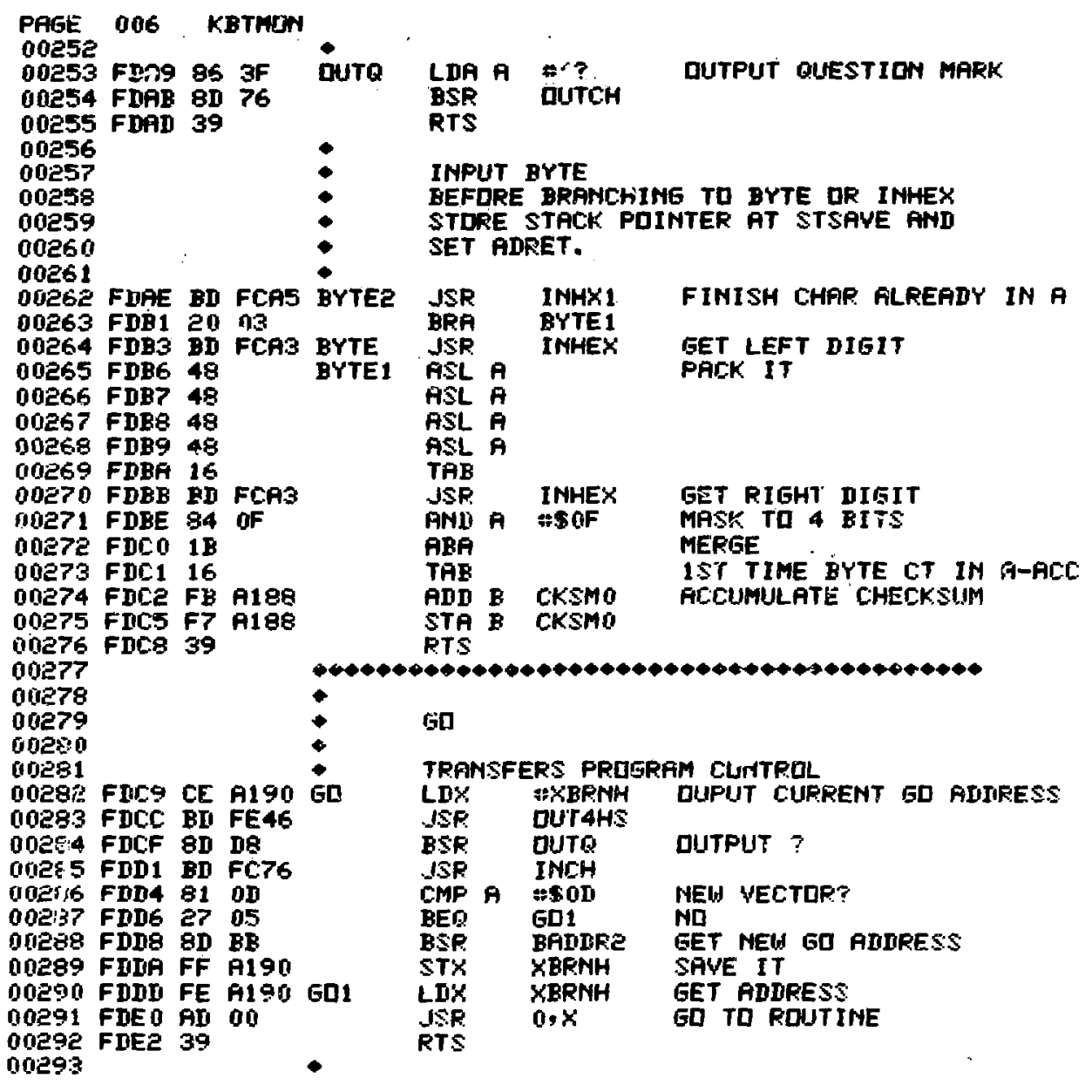




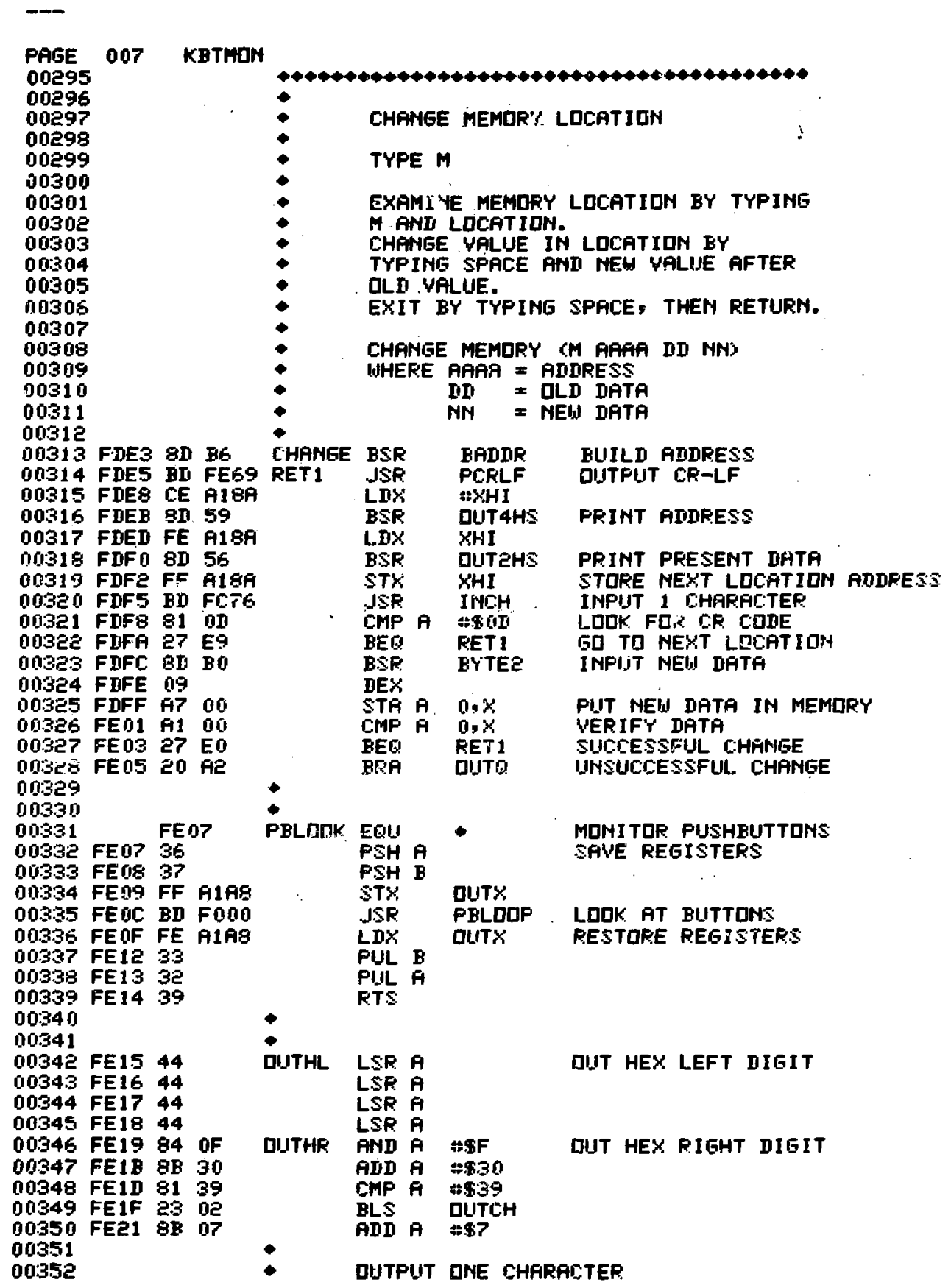




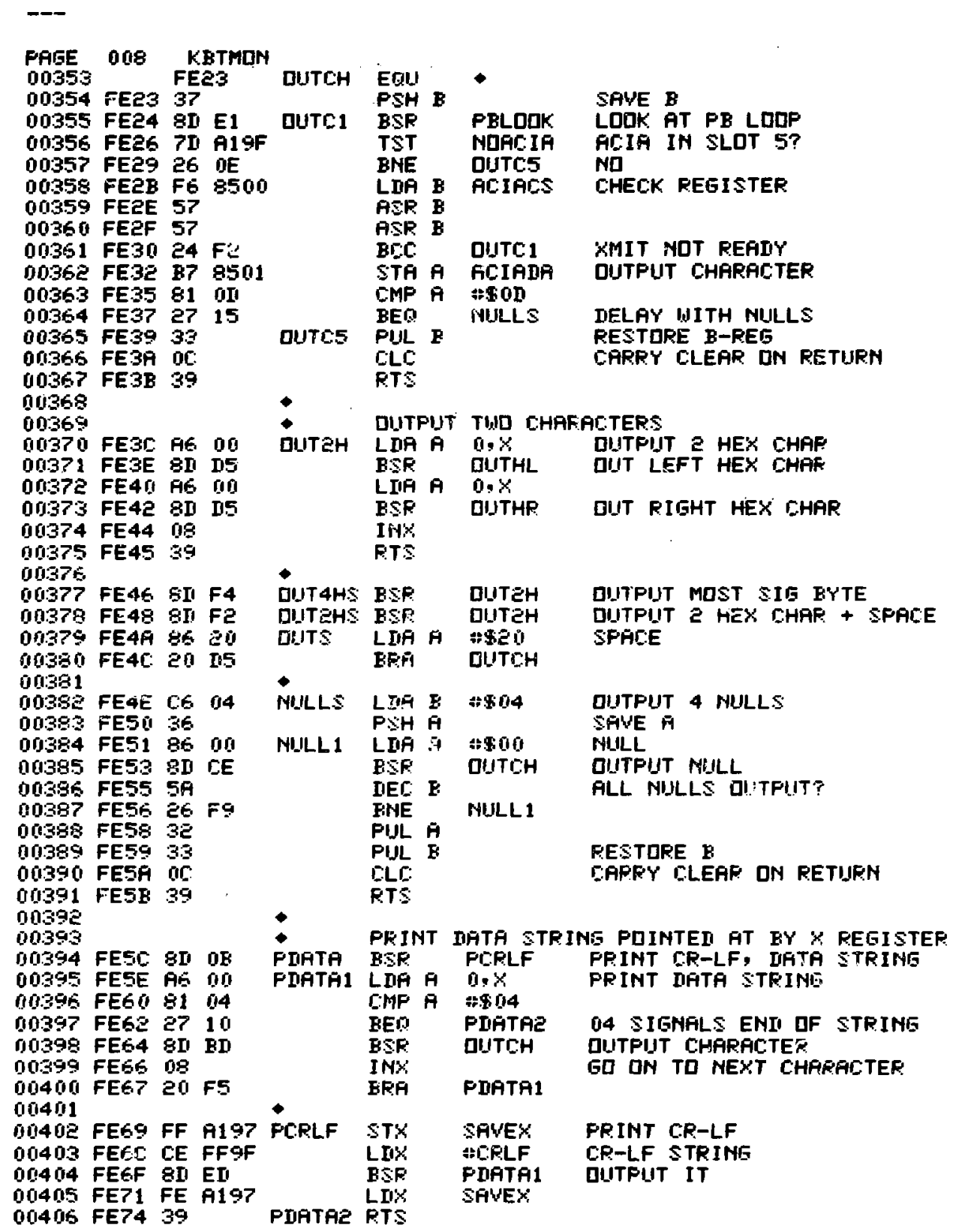




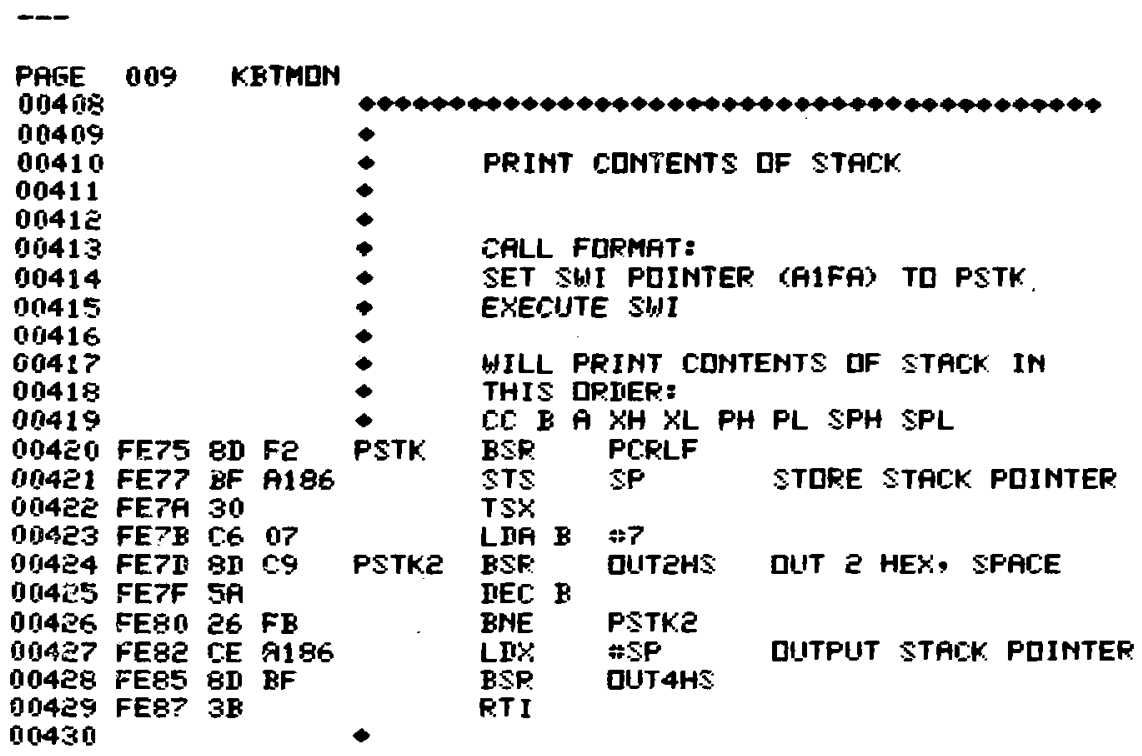




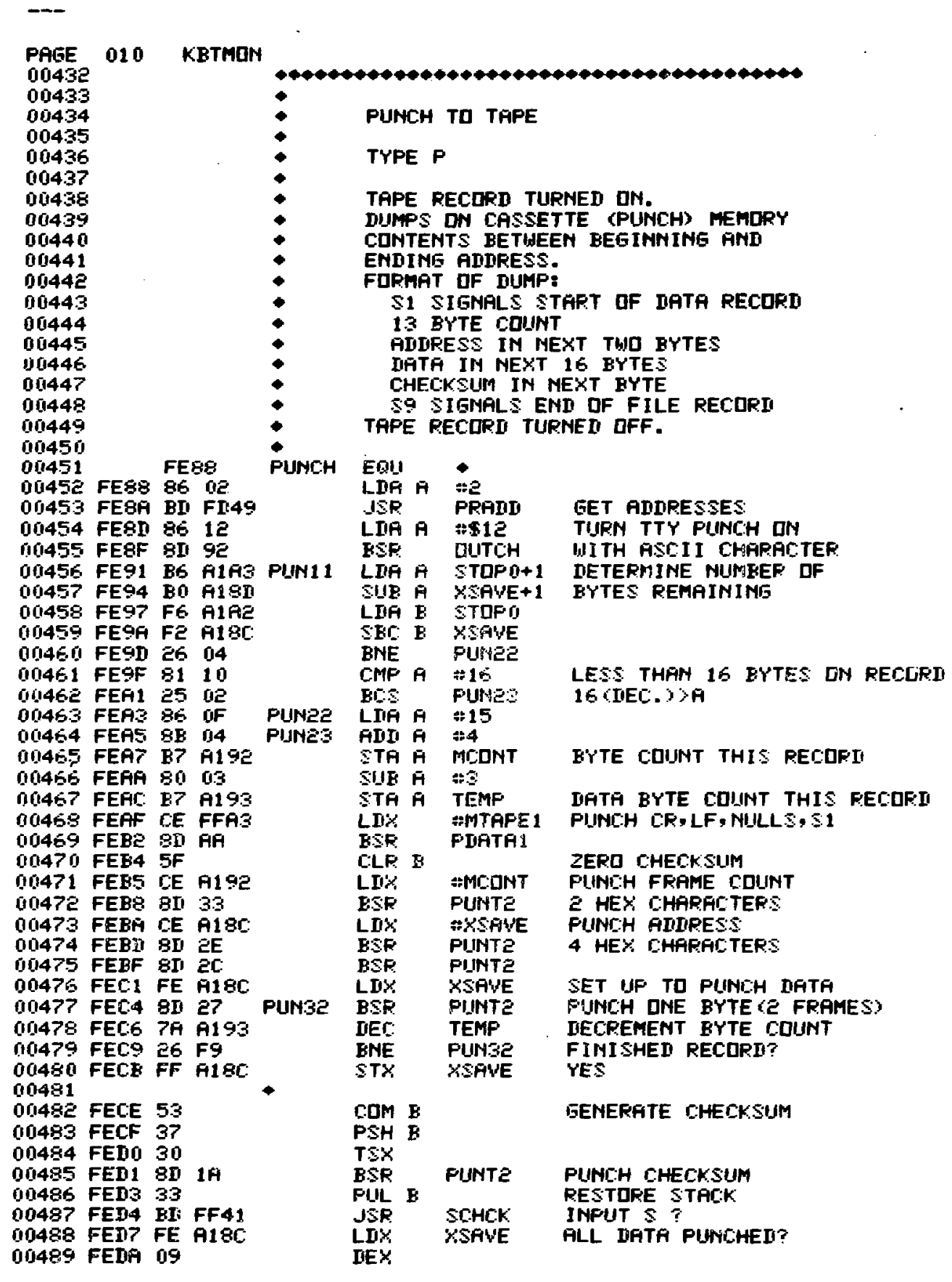




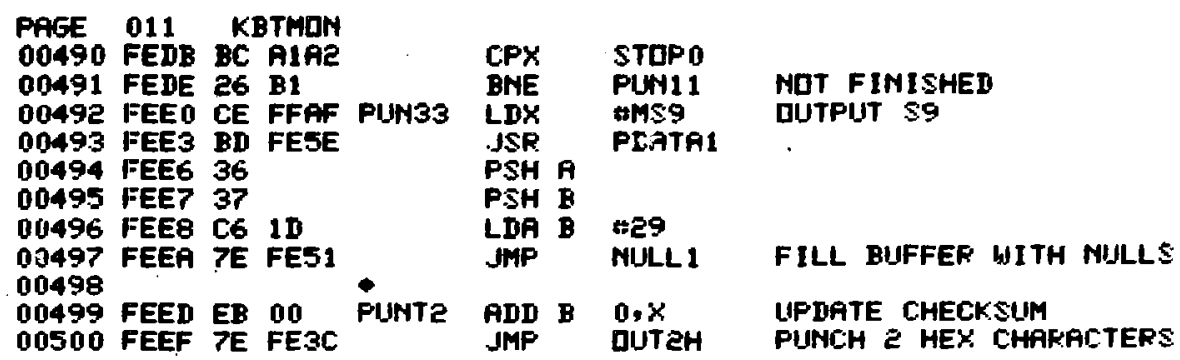

00493 FEE3 BD FESE JSR PEATA1

PSH A

PSH $\mathrm{B}$

$\operatorname{LDA} B=29$

JMP NULL 1

PUNTz aDD $B$ o,X JMF DUTEH

NOT FIMISHED

DUTPUT $\$ 9$

FILL BUFFER WITH MULLS LIFDATE CHECKSUM PUNCH 2 HEX CHARACTERS 


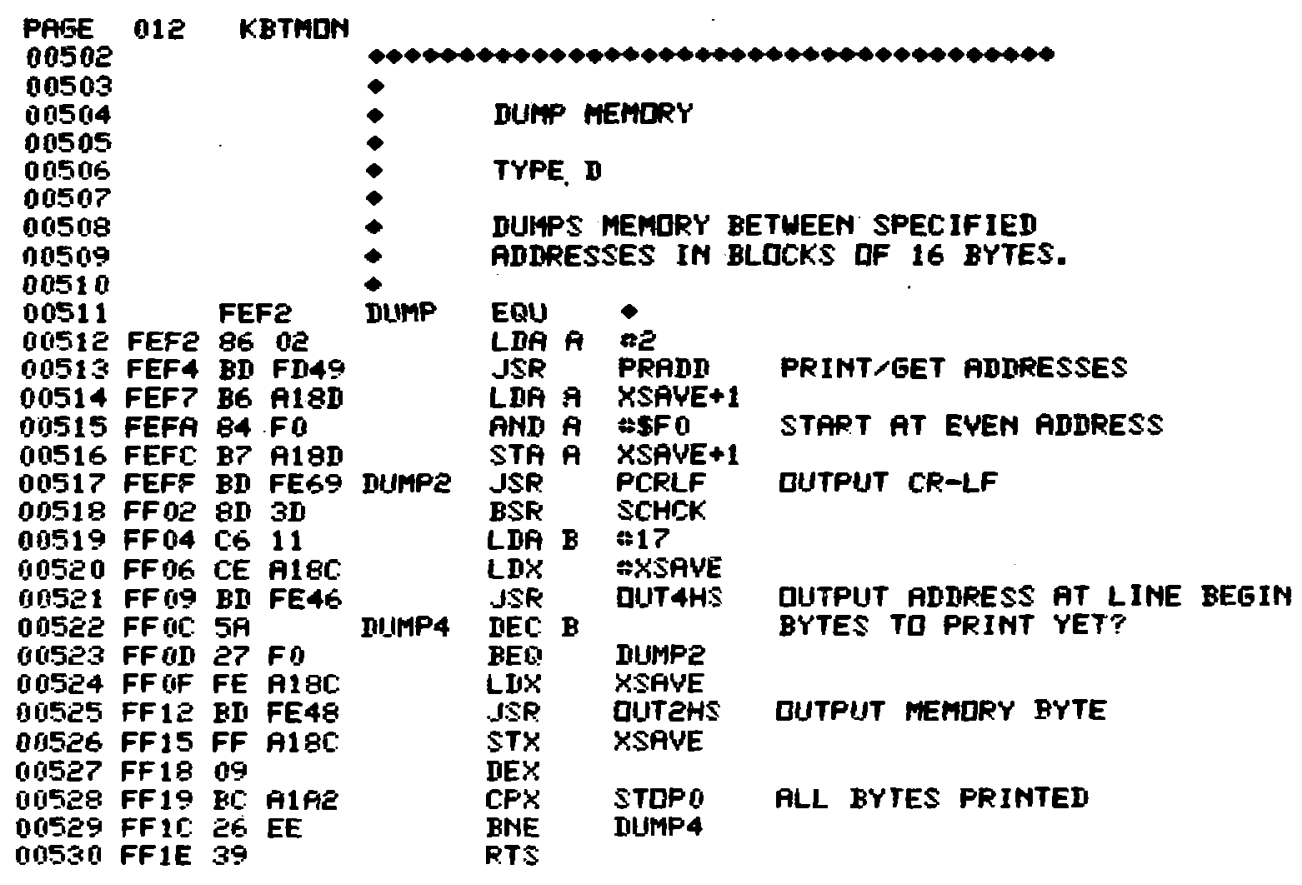




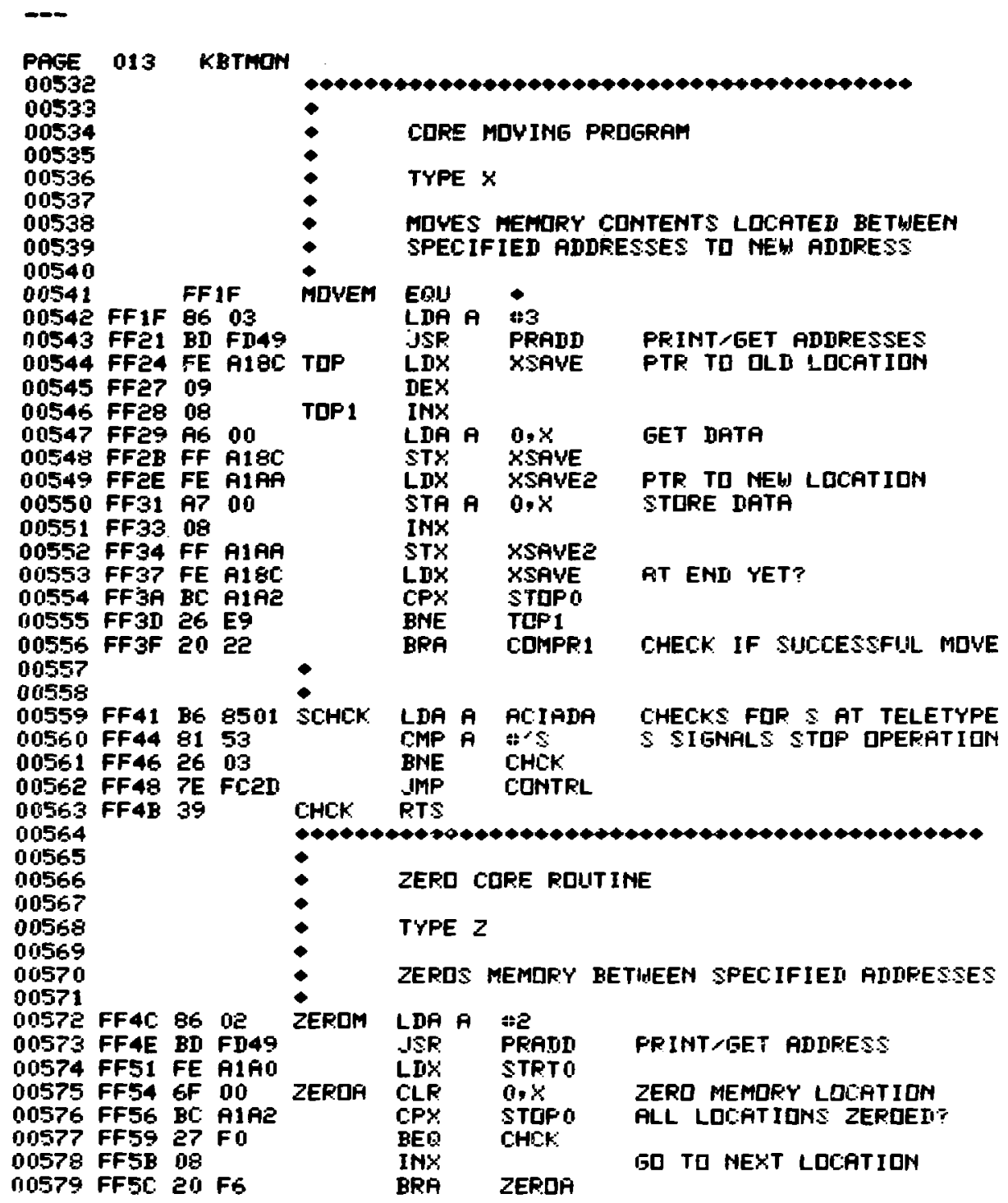




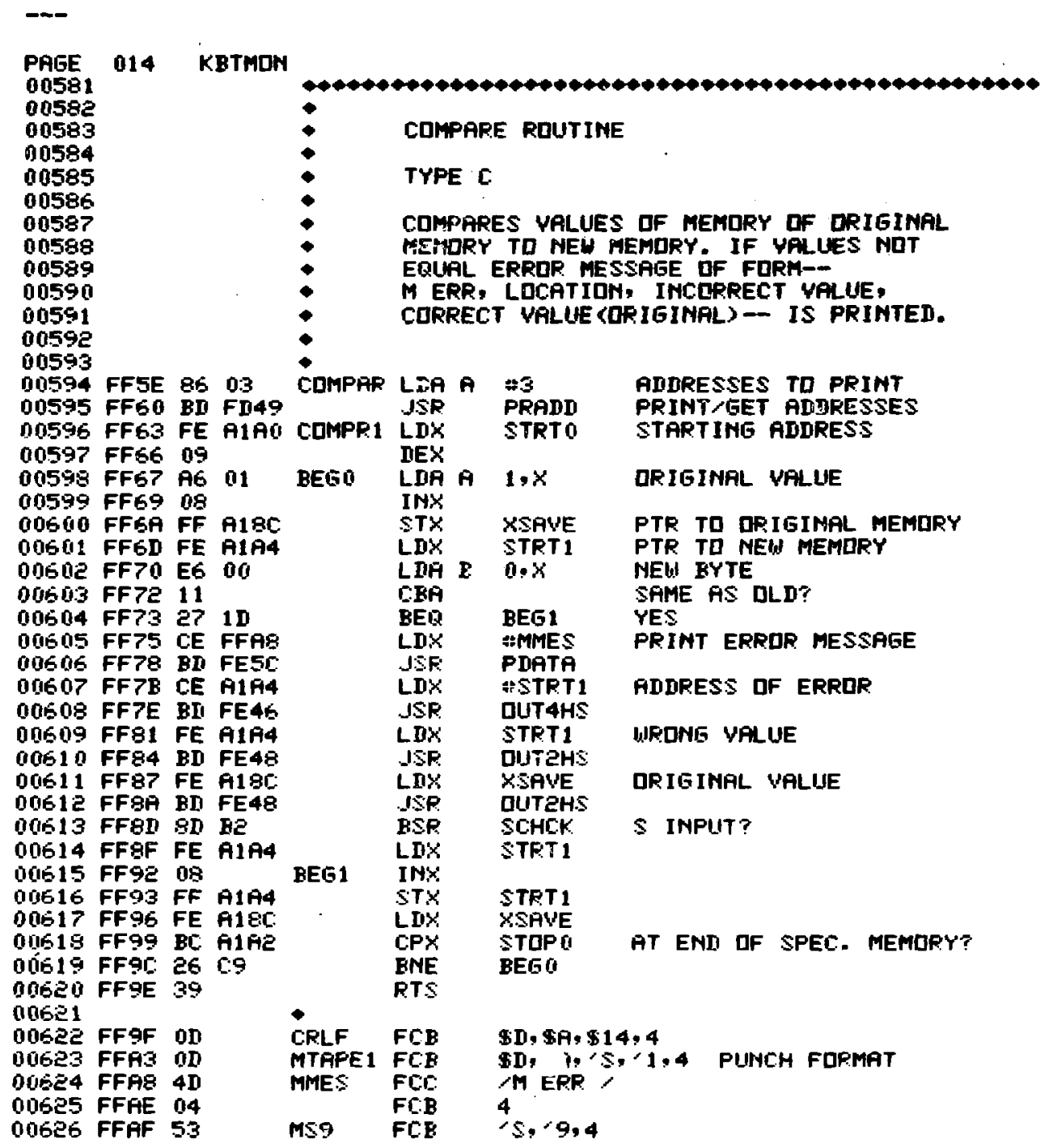




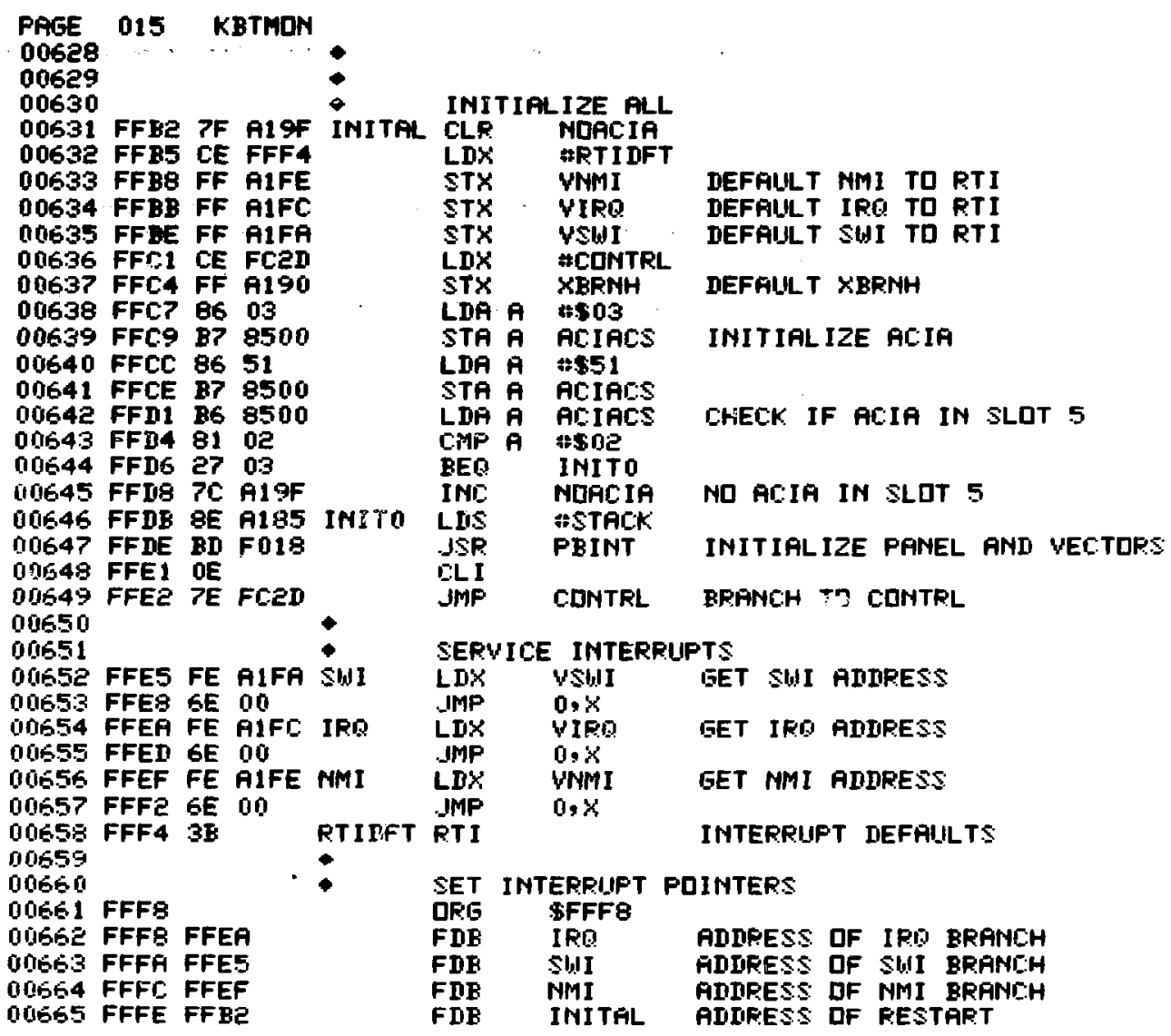




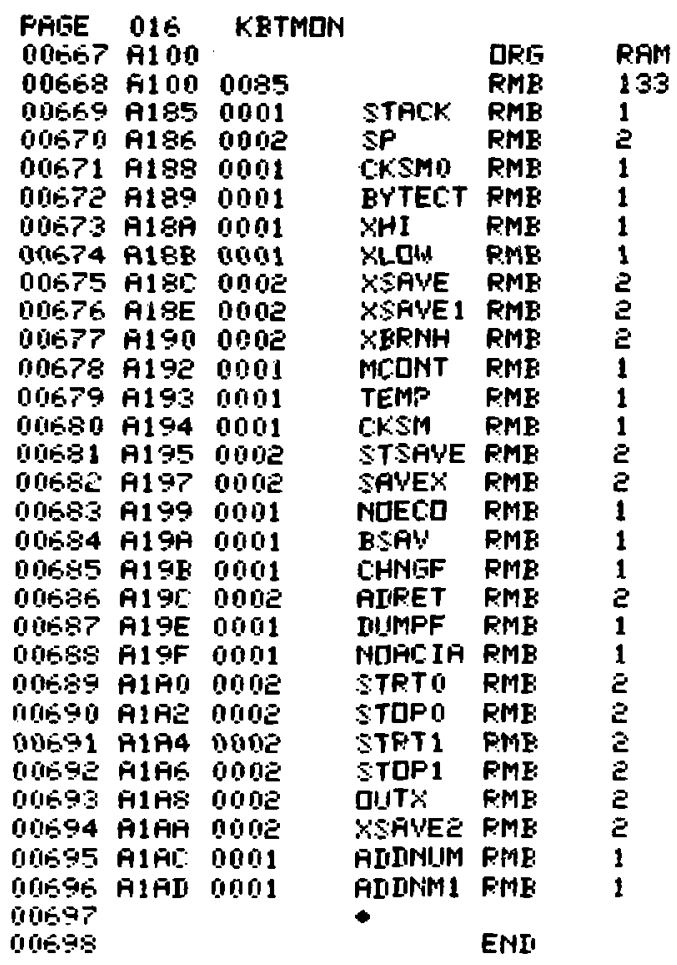

STACK

STAEK PDINTER

CHECKSUM + CKSM

BYTE COUNT

XREG HIBH

XPEG LQW

SRVE $X$ REGISTER

SHVE $X$ REGISTER

150 BRANCH

MAXI MUM CDUNT

TEMPQRARY CDUNT

CHECKSUM

STALK SAWE

SANE $X$ REGISTER.

ND ECHD FLAIS

SAWE $B$ FICE:

RELDCATABLE LDAI FLAE

RETURN AIDRESS:

DIIMP FLAG

SIGNALS IF AEIA IN SLDT 5

AIIIF DF PEM TO RE MDVEI

ENI AIIIP DF FGM TO RE MUYEII

NEW BEGIMTHIMIS AIIIRESS

NEU ENIIING RDIRESS

TEMF $X$ STDFAGE FDR FRLDDK

SAVE $X$ FEIG

NUMBER DF FIIIRESSES TO BET

TEMF, STORAIEE OF AIIIHIM

TDTAL EFROFS DUOMOI 


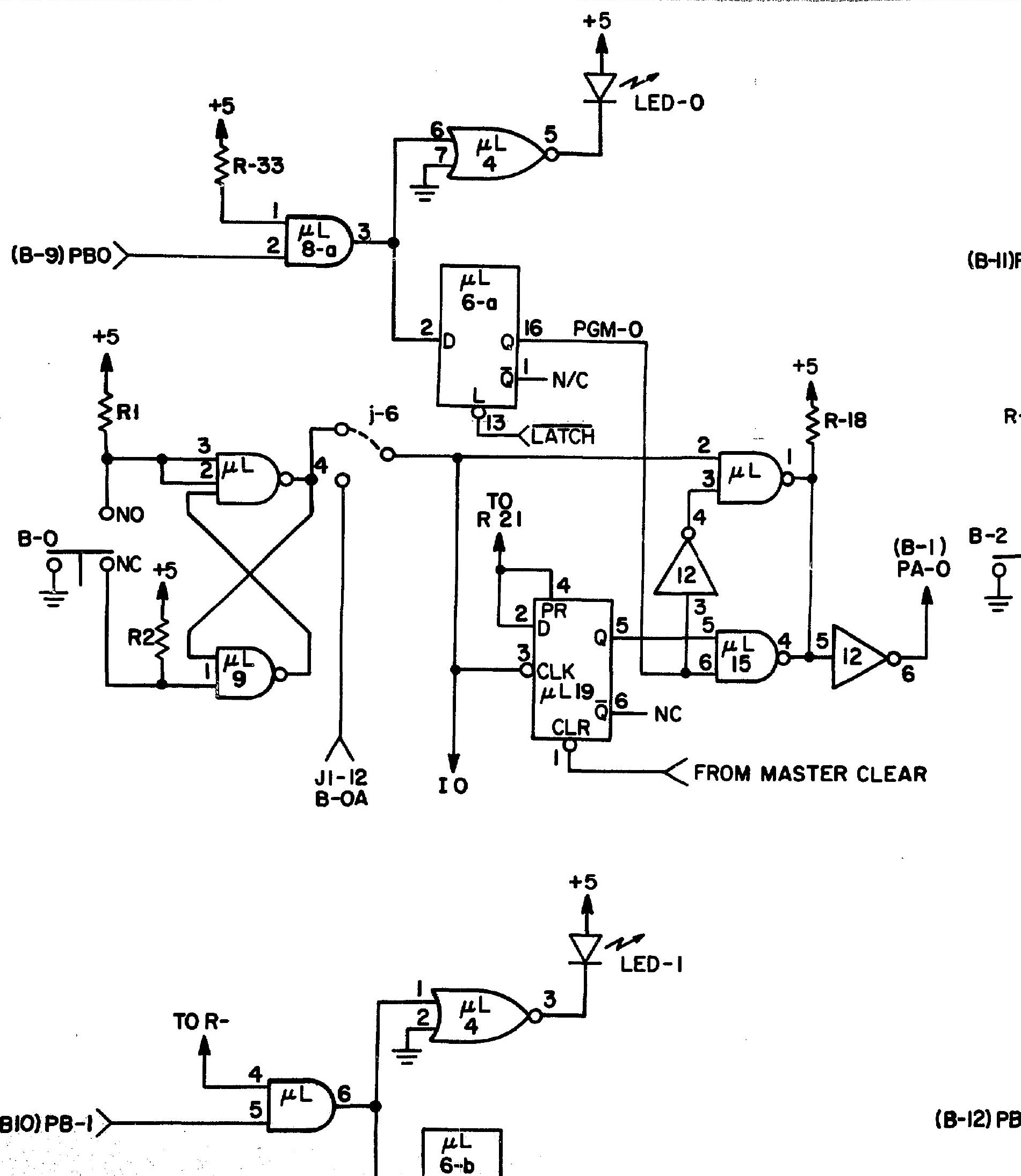




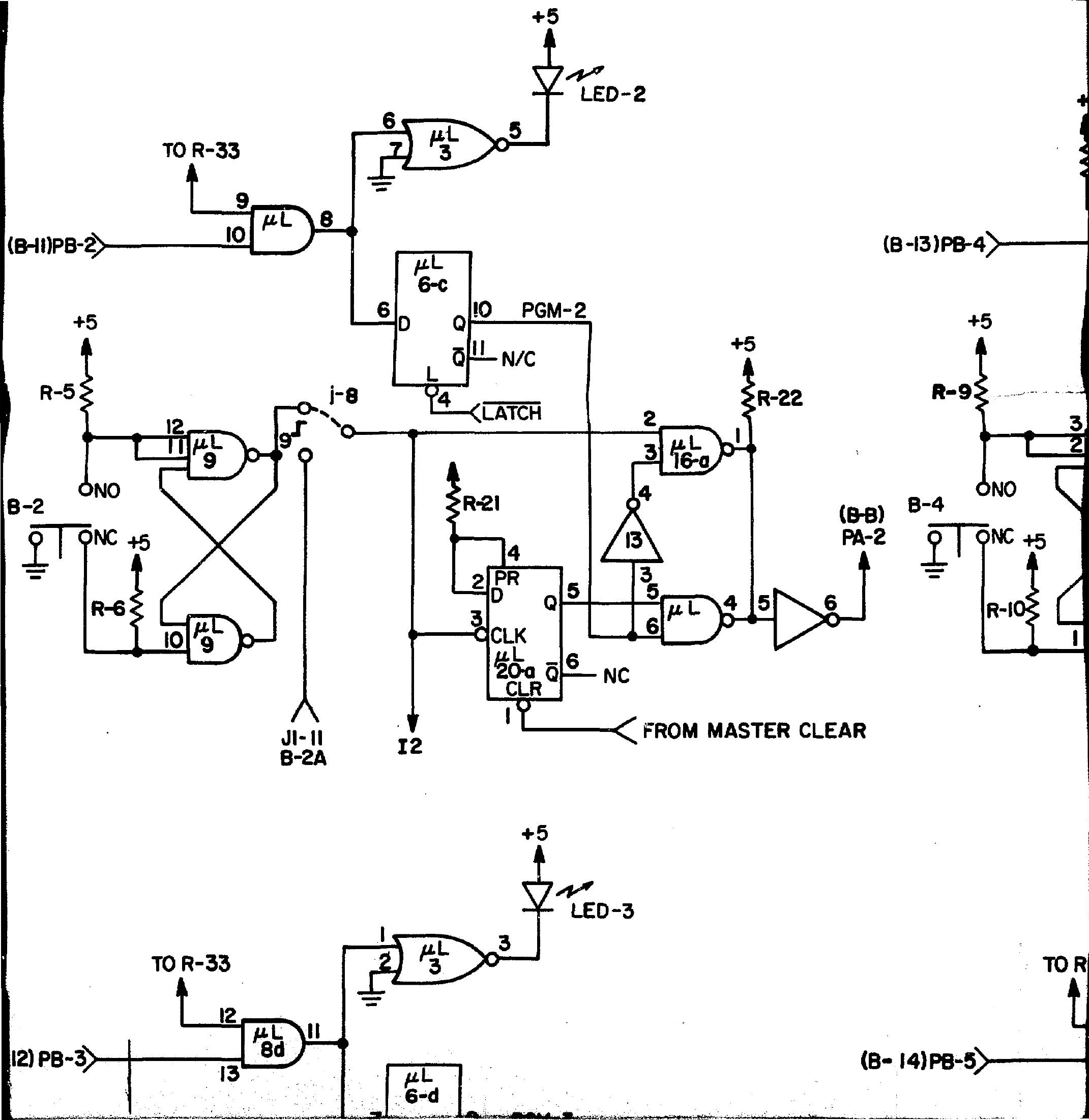





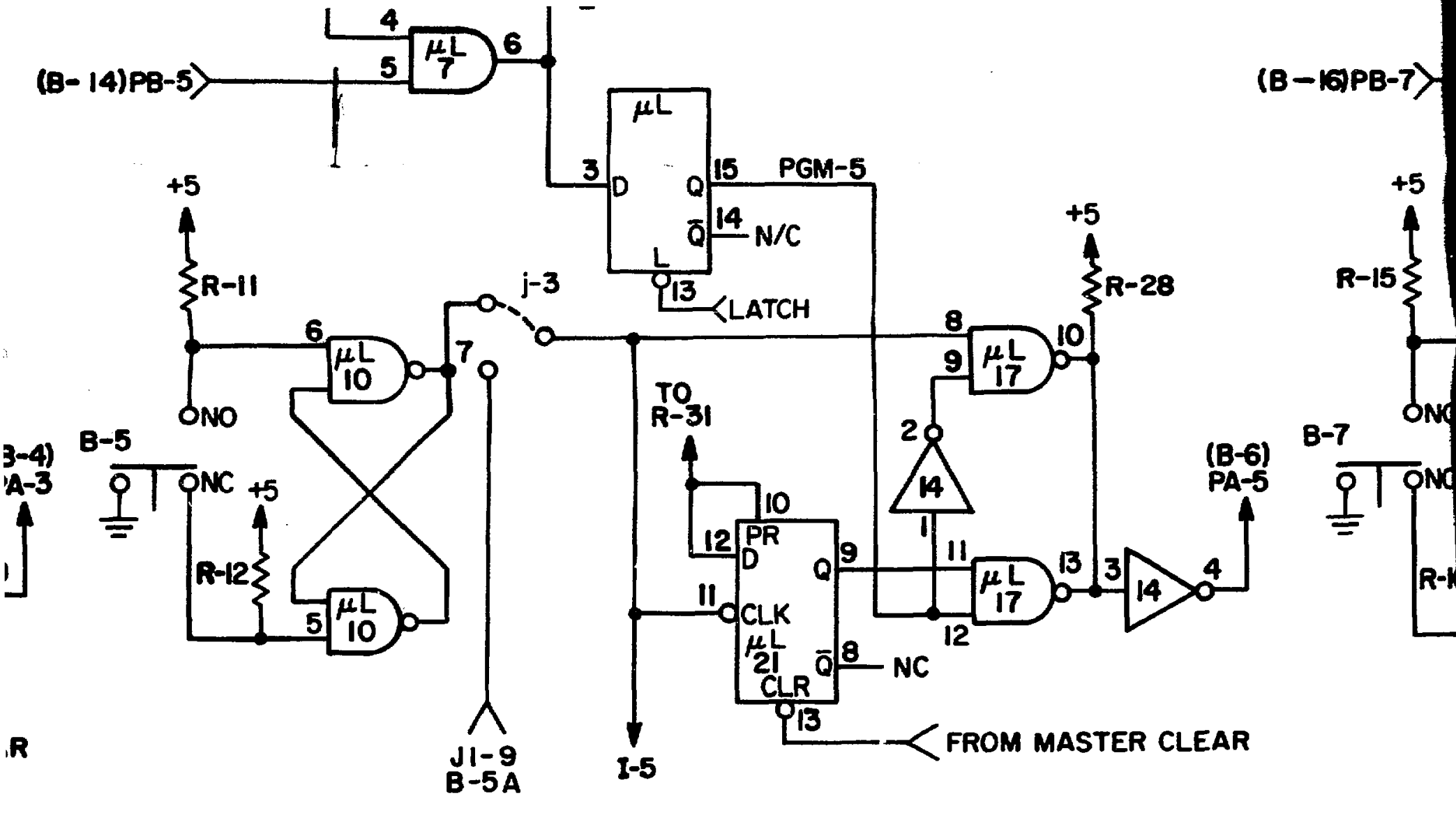

\section{NOTES:}

UL's 1,2,3 \& 4; 75454

UL $5 \& 6 ; 7475$

UL 7,8 \& 11; 7408

UL $9 \& 10 ; 74279$

UL 12,13 \& 14; 7404

UL 15,16,17 \& 18; 740 ?

UL 19,20,21 \& 22; 7474

UL 23; 7433

ALL R's: $3.3 \mathrm{~K}$ 1/4 WATT

LED's 0 THRU 7: HP-5082 


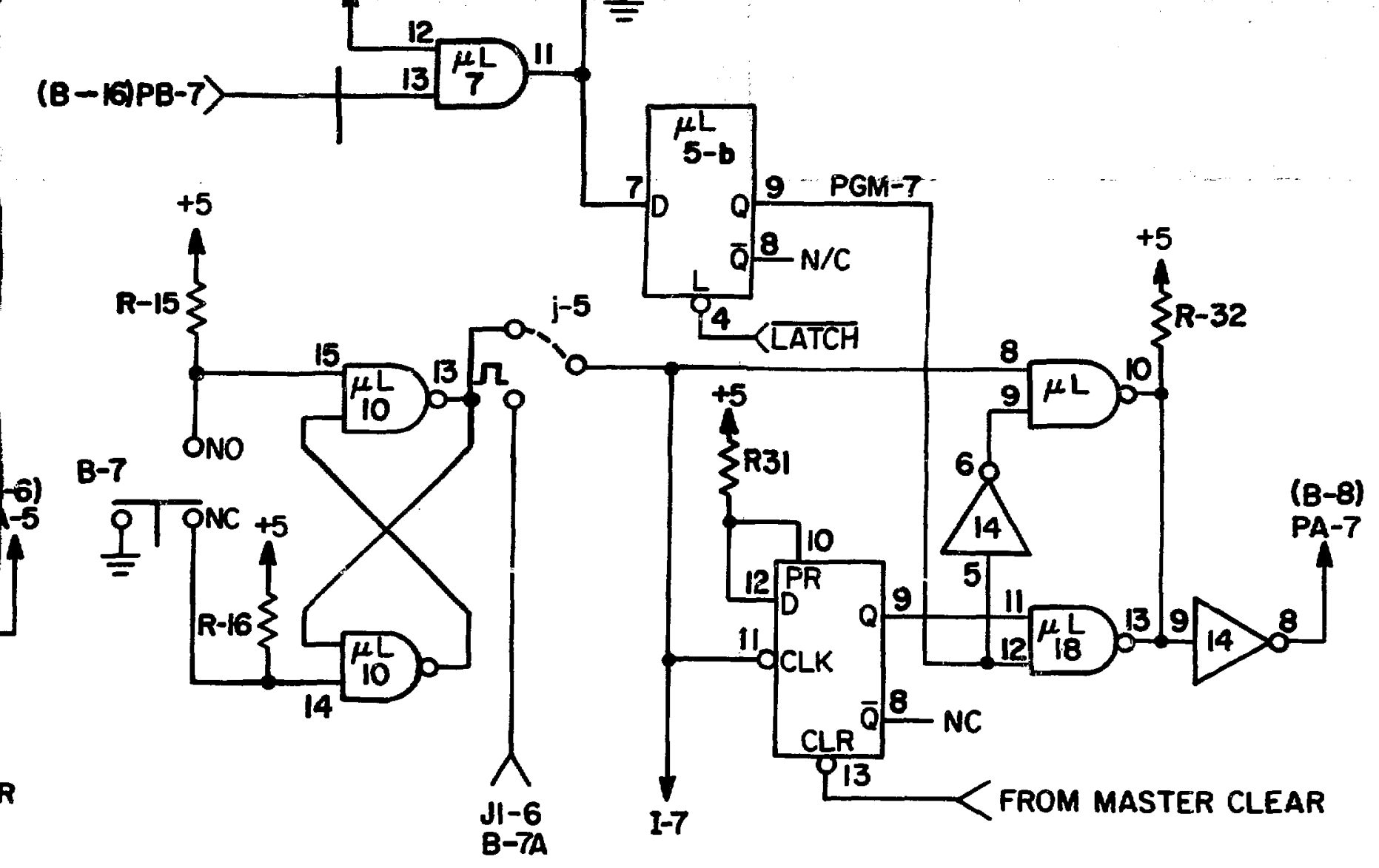

